University of Arkansas, Fayetteville

ScholarWorks@UARK

Graduate Theses and Dissertations

8-2018

\title{
Closed Range Composition Operators on BMOA
}

\author{
Kevser Erdem \\ University of Arkansas, Fayetteville
}

Follow this and additional works at: https://scholarworks.uark.edu/etd

Part of the Numerical Analysis and Computation Commons

Citation

Erdem, K. (2018). Closed Range Composition Operators on BMOA. Graduate Theses and Dissertations Retrieved from https://scholarworks.uark.edu/etd/2831

This Dissertation is brought to you for free and open access by ScholarWorks@UARK. It has been accepted for inclusion in Graduate Theses and Dissertations by an authorized administrator of ScholarWorks@UARK. For more information, please contact scholar@uark.edu. 
Closed range composition operators on BMOA

A dissertation submitted in partial fulfillment of the requirements for the degree of Doctor of Philosophy in Mathematics

by

\author{
Kevser Erdem \\ Nigde University \\ Bachelor of Science in Mathematics, 2006 \\ University of Arkansas \\ Master of Science in Mathematics, 2015
}

August 2018

University of Arkansas

This dissertation is approved for recommendation to the Graduate Council.

Maria Tjani, Ph.D.

Dissertation Director

Phil Harrington, Ph.D.

Committee Member
Daniel Luecking, Ph.D.

Committee Member 


\begin{abstract}
Let $\varphi$ be an analytic self-map of the unit disk $\mathbb{D}$. The composition operator with symbol $\varphi$ is denoted by $C_{\varphi}$. Reverse Carleson type conditions, counting functions and sampling sets are important tools to give a complete characterization of closed range composition operators on $B M O A$ and on $Q_{p}$ for all $p \in(0, \infty)$.

Let $\mathcal{B}$ denote the Bloch space, let $H^{2}$ denote the Hardy space. We show that if $C_{\varphi}$ is closed range on $\mathcal{B}$ or on $H^{2}$ then it is also closed range on $B M O A$. Closed range composition operators $C_{\varphi}: \mathcal{B} \rightarrow B M O A$ are also characterized. Laitila found the isometries among composition operators on $B M O A$. We extend this to $Q_{p}$ for all $p \in(0, \infty)$
\end{abstract}




\section{Acknowledgements}

I would like to take this opportunity to express my gratitude to my mentor and advisor Dr. Maria Tjani, for her time, persistence, support, motivation. $\Sigma \alpha \varsigma \varepsilon v \chi \alpha \rho \iota \sigma \tau \omega \pi o \lambda v$ -thank you so much- Dr. Tjani for always sticking with and showing the positive side of things; your help with improving myself. I cannot thank you enough for your mentoring, positivity, and optimism. You are the best.

I also would like to extend my appreciation to the rest of the committee: Dr. Daniel H. Luecking and Dr. Phil Harrington for their time and support during my studies. I would like to thank the department chair Dr. Mark Johnson for his care when he arranges my teaching schedule and for his referrals during my job applications. I would like to thank Dr. John Akeroyd for his job referrals, as well as Dr. Matt Clay for his guidance and referrals during my job applications.

I would like to express my deepest gratitude to my mother Salise Baktir and father Hamdi Baktir; my siblings Mehmet, Beyza, and Betul; as well as my in-laws Hatice and Bekir Erdem. Throughout my whole education history, you have been so kind and supportive. You were with me when needed. Thank you so much for everything (Iyiki varsiniz!).

I do not want to forget my dear friend Nurhan for her support and numerous other friends and colleagues at the U of A, such as Daniel, Melinda, Rachael, and Razieh whose presence contributed to enjoying the graduate school and relieved daily stresses.

Last but not least, I would like to recognize the staff at the Department of Mathematical Sciences at the University of Arkansas who have been very friendly and helpful at all matters. 


\section{Dedication}

I dedicate this dissertation to my beloved daughter Afra and husband Burhan. They have a special place in my completion of this degree. A huge thank you for your support behind the scenes. You are nothing but a blessing. Thank you darlings, for always being with me and believing in success; without your positive energy and humongous smiles I would not be standing where I am now. 


\section{Table of Contents}

1 Introduction $\quad 1$

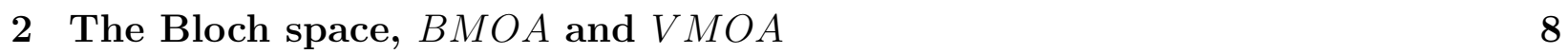

3 Bounded and closed range composition operators on $B M O A \quad 19$

4 Composition operators on $Q_{p} \quad 42$

$\begin{array}{lc}\text { References } & 54\end{array}$ 


\section{Introduction}

The Hardy space $H^{2}$ is the collection of analytic functions on the open unit disk $\mathbb{D}$ whose power series have square summable coefficients, that is if

$$
f(z)=\sum_{n=0}^{\infty} a_{n} z^{n}
$$

then

$$
\|f\|_{H^{2}}^{2}=\sum_{n=0}^{\infty}\left|a_{n}\right|^{2}<\infty .
$$

The above defines a norm in $H^{2}$. Let $\varphi$ be an analytic self-map of $\mathbb{D}$. The composition operator, denoted by $C_{\varphi}$, is the operator that maps $f$ to $f \circ \varphi$, that is

$$
C_{\varphi} f=f \circ \varphi
$$

for $f$ analytic on $\mathbb{D}$. Studies of composition operators start with the Hardy space setting. Boundedness, compactness as well as closed rangeness have been studied in this setting. Then, a very natural question is to study these properties on other function spaces.

Closed range composition operators were first studied by Cima, Thomson and Wogen in [9], in the context of $H^{2}$. Their results are in terms of the boundary behavior of the symbol $\varphi$. They posed the question of studying closed range composition operators in terms of properties of $\varphi$ on $\mathbb{D}$ rather than on its boundary. The question was answered by Zorboska in [38] who studied the problem in $H^{2}$ and also in weighted Bergman spaces. In [17] Jovovic and MacCluer studied the problem in weighted Dirichlet spaces. Closed range composition operators in the Bloch space were investigated by Ghatage, Yan and Zheng in [14], by Ghatage, Zheng and Zorboska in [15], by Chen and Gauthier in [8] and by Akeroyd, Ghatage and Tjani in [3]. Akeroyd, Ghatage and Tjani revisited the problem in the context of the Bergman space, see [2] and [3]. The journey on closed range composition 
operators is continued on Besov type spaces $\mathcal{B}_{p, \alpha}, p>1, \alpha>1$ by Tjani, see [34].

Let $X, Y$ be Banach spaces, let $T: X \rightarrow Y$ be a linear operator. The norms in $X, Y$ are denoted by $\|.\|_{X},\|.\|_{Y}$ respectively. We say that a bounded operator $T$ is bounded below if there exists a $\delta>0$ such that for all $x \in X$

$$
\|T x\|_{Y} \geq \delta\|x\|_{X}
$$

Moreover, if $T X$ is a closed subspace of $Y$, then we say that $T$ is closed range.

The Closed Graph Theorem, see [37, Theorem 1.5], says that $T$ is a bounded operator if and only if its graph is closed in $X \times Y$. If $T$ is one-to-one and is closed range then, by the Closed Graph Theorem and since $T^{-1}: T(X) \rightarrow X$ is a bounded operator, and we can easily see that $T$ is bounded below. Moreover, if $T$ is bounded below and $\left(x_{n}\right)$ is a sequence in $X$ such that $T x_{n} \rightarrow y$ for some $y \in Y$, then by (1) we can easily see that $\left(x_{n}\right)$ is a Cauchy sequence, hence it converges to some $x \in X$. Since $T$ is a bounded operator $T x_{n} \rightarrow T x$ and so $T x=y$. We conclude that $T$ is closed range. Therefore if $T: X \rightarrow Y$ is a bounded linear operator that is one-to-one then $T$ is closed range if and only if $T$ is bounded below.

Here and thereafter, if $A$ and $B$ are two quantities that depend on an analytic function $f$ on $\mathbb{D}$, we will use the notation $A \asymp B$ and say that $A$ is equivalent to $B$; that is, there exist positive constants $c_{1}, c_{2}$ such that

$$
c_{1} A \leq B \leq c_{2} A
$$

This thesis aims to contribute by giving a complete characterization of closed range composition operators on $B M O A$, the space of analytic functions on $\mathbb{D}$ with bounded mean oscillation and also on the spaces $Q_{p}, 0<p<\infty$.

In Chapter 2, we give definitions of $B M O A, V M O A$, the Bloch Space, the Dirichlet space and the Bergman space. We also give the properties and connections of these spaces that we will use in later chapters. 
In Chapter 3, we characterize closed range composition operators, $C_{\varphi}$, on $B M O A$. It is well known that composition operators in $B M O A$ are bounded for any symbol $\varphi$ that is an analytic self-map of $\mathbb{D}$. A crucial tool to this work is a non univalent change of variables formula involving the counting function for $B M O A$ that is similar to the well known change of variables formula involving the Nevanlinna counting function. Let $q \in \mathbb{D}$, $\alpha_{q}=\frac{q-z}{1-\bar{q} z}$, and $\zeta \in \mathbb{D}$. If $\zeta \in \varphi(\mathbb{D})$ the $B M O A$ counting function is

$$
N_{q, \varphi}(\zeta)=\sum_{\varphi(z)=\zeta}\left(1-\left|\alpha_{q}(z)\right|^{2}\right)
$$

it is understood that if $\zeta$ is not in the range of $\varphi$, then $N_{q, \varphi}(\zeta)=0$.

Given $\varepsilon>0$ and $q \in \mathbb{D}$ let $G_{\varepsilon, q}=\left\{\zeta: N_{q, \varphi}(\zeta)>\varepsilon\left(1-\left|\alpha_{q}(\zeta)\right|^{2}\right)\right\}$ and let $G_{\varepsilon}=\cup_{q \in \mathbb{D}} G_{\varepsilon, q}$. We define the notion of sampling in $B M O A$ and prove the following.

Theorem 3.6 Let $\varphi$ be a non-constant analytic self map of $\mathbb{D}$. If $C_{\varphi}$ is closed range on $B M O A$ then there exists $\varepsilon>0$ such that $G_{\varepsilon}$ is a sampling set for BMOA. Moreover, if $\cap_{q \in \mathbb{D}} G_{\varepsilon, q}$ is a sampling set for $B M O A$, then $C_{\varphi}$ is closed range on BMOA.

The above result provides necessary and in general different sufficient conditions for $C_{\varphi}$ to have closed range on $B M O A$. Next we look for a single condition that is both necessary and sufficient.

Given $\varepsilon>0$ and $q, q^{\prime} \in \mathbb{D}$ let $G_{\varepsilon, q^{\prime}, q}=\left\{\zeta: N_{q^{\prime}, \varphi}(\zeta)>\varepsilon\left(1-\left|\alpha_{q}(\zeta)\right|^{2}\right)\right\}$.

Theorem 3.8 Let $\varphi$ be an analytic self-map of $\mathbb{D}$. Then, there exists $k>0$ such that for all $q \in \mathbb{D},\left\|\alpha_{q} \circ \varphi\right\|_{*} \geq k$ if and only if there exists an $\varepsilon>0$ and $r \in(0,1)$ such that for all $q \in \mathbb{D}$, there exists $q^{\prime} \in \mathbb{D}$ such that

$$
\frac{\left|G_{\varepsilon, q^{\prime}, q} \cap D(q, r)\right|}{|D(q, r)|} \asymp 1 .
$$

Moreover, $G_{\varepsilon}=\cup_{q \in \mathbb{D}} G_{\varepsilon, q^{\prime}, q}$ satisfies the reverse Carleson condition.

By using reverse Carleson type sets we prove the following.

Theorem 3.11 Let $\varphi$ be an analytic self-map of $\mathbb{D}$. Then the composition operator 
$C_{\varphi}: \mathcal{B} \rightarrow B M O A$ is closed range if and only if there exists an $\varepsilon>0$ and $r \in(0,1)$ such that for all $q \in \mathbb{D}$, there exists $q^{\prime} \in \mathbb{D}$ such that

$$
\frac{\left|G_{\varepsilon, q^{\prime}, q} \cap D(q, r)\right|}{|D(q, r)|} \asymp 1 .
$$

In Theorem 3.14 we show that a set satisfies the reverse Carleson condition if and only if it is a sampling set on $B M O A$.

Next, we use another method and provide a full characterization of closed range composition operators on $B M O A$. We note that by Theorem 3.8 and Theorem 3.11, $C_{\varphi}$ is closed range on $B M O A$ if and only if it is closed range as an operator from $\mathcal{B}$ to $B M O A$.

Theorem 3.12 The composition operator $C_{\varphi}$ is closed range on BMOA if and only if there exists a constant $k>0$ such that $\left\|\alpha_{q} \circ \varphi\right\|_{*} \geq k$, for all $q \in \mathbb{D}$.

Since a composition operator $C_{\varphi}$ is bounded on $V M O A$ if and only if $\varphi \in V M O A$, we have the following.

Corollary 3.13 Let $\varphi \in V M O A$. Then $C_{\varphi}$ is closed range on VMOA if and only if there exists a constant $k>0$ such that $\left\|\alpha_{q} \circ \varphi\right\|_{*} \geq k$, for all $q \in \mathbb{D}$.

We also show that if $C_{\varphi}$ is closed range on the Bloch space or the Hardy space then it is closed range on BMOA, see Corollary 3.16 and Corollary 3.18. Moreover, we show that for a univalent self-map of $\mathbb{D}, C_{\varphi}$ is closed range on $\mathcal{B}$ if and only if $C_{\varphi}$ closed range on $B M O A$, see Corollary 3.17 .

Finally, in Theorem 3.19 we give another characterization of closed range composition operators on $B M O A$.

Theorem 3.19 For each $\varphi$ analytic self map of $\mathbb{D}$, the following conditions are equivalent:

(a) There exists $k \in(0,1]$ such that for every $w \in \mathbb{D},\left\|\alpha_{w} \circ \varphi\right\|_{*} \geq k$.

(b) There exists $k \in(0,1]$ such that for every $w \in \mathbb{D}$ there exists $w^{\prime} \in \mathbb{D}$ with 
$\left|\alpha_{w}\left(w^{\prime}\right)\right|^{2} \leq 1-k^{2}$, there exists a sequence $\left(q_{n}\right)$ in $\mathbb{D}$ such that $\varphi\left(q_{n}\right) \rightarrow w^{\prime}$ and

$$
\lim _{n \rightarrow \infty}\left\|\varphi_{q_{n}}\right\|_{H^{2}} \geq k
$$

where for all natural numbers $n, \varphi_{q_{n}}=\alpha_{\varphi\left(q_{n}\right)} \circ \varphi \circ \alpha_{q_{n}}$.

In Chapter 4 we define the spaces $Q_{p}$ and study closed range composition operators on them.

Firstly, we show a Lipschitz continuity property that $\left\|f \circ \alpha_{q}-f(q)\right\|_{Q_{p}}$ has with respect to the pseudohyperbolic metric. The space $\mathcal{D}_{p}$ will be defined in Chapter 4 .

Theorem 4.1 Let $p \in(0,1]$. Then, for each $f \in Q_{p}$ and for all $q, w \in \mathbb{D}$

$$
\left|\left\|f \circ \alpha_{q}-f(q)\right\|_{\mathcal{D}_{p}}^{2}-\left\|f \circ \alpha_{w}-f(w)\right\|_{\mathcal{D}_{p}}^{2}\right| \leq 2^{p+1} \rho(q, w)\|f\|_{Q_{p}}^{2} .
$$

If $p \in(1, \infty)$ then $Q_{p}$ is the Bloch space with and equivalent norm and we have the following.

Theorem 4.3 Let $p \in(1, \infty)$. Then for each $f \in Q_{p}$ and all $q, w \in \mathbb{D}$

$$
\left|\left\|f \circ \alpha_{q}-f(q)\right\|_{\mathcal{D}_{p}}^{2}-\left\|f \circ \alpha_{w}-f(w)\right\|_{\mathcal{D}_{p}}^{2}\right| \leq 4 p \rho(q, w)\|f\|_{Q_{p}}^{2}
$$

Let $p \in[0, \infty)$. The counting function for $Q_{p}$ for each $q \in \mathbb{D}$, and if $\zeta \in \varphi(\mathbb{D})$ is defined by

$$
N_{q, \varphi}(\zeta, p)=\sum_{\varphi(z)=\zeta}\left(1-\left|\alpha_{q}(z)\right|^{2}\right)^{p}
$$

if $\zeta \notin \varphi(\mathbb{D})$ then $N_{q, \varphi}(\zeta, p)=0$.

In Theorem 4.5 and Theorem 4.6 we give necessary and different sufficient conditions for $C_{\varphi}$ to be closed range in $Q_{p}$, for $p \in(0,1)$, in terms of sampling type measures.

Theorem 4.5 Let $p \in(0,1)$ and assume that $C_{\varphi}$ is a bounded operator on $Q_{p}$. If there 
exists an $\epsilon>0$ such that

$$
\sup _{q \in \mathbb{D}} \int_{\cap_{q \in \mathbb{D}} G_{\epsilon, q}}\left|f^{\prime}(\zeta)\right|^{2} N_{q, \varphi}(\zeta) d A(\zeta) \asymp\|f\|_{Q_{p}}^{2}
$$

then the composition operator $C_{\varphi}$ is closed range on $Q_{p}$.

Theorem 4.6 Let $p \in(0,1)$ and assume that $C_{\varphi}$ is a bounded operator on $Q_{p}$. If $C_{\varphi}$ is closed range on $Q_{p}$ then there exists an $\epsilon>0$ such that for all $f \in Q_{p}$

$$
\sup _{q \in \mathbb{D}} \int_{G_{\epsilon}}\left|f^{\prime}(\zeta)\right|^{2} N_{q, \varphi}(\zeta) d A(\zeta) \asymp\|f\|_{Q_{p}}^{2}
$$

The main results of the chapter are Theorem 4.7, Theorem 4.8 and Theorem 4.9. They provide a complete characterization of closed range composition operators on $Q_{p}$ for all $p \in(0, \infty)$. We also note that Theorem 4.9 gives a characterization of all isometries on $Q_{p}$ for all $p \in(0, \infty)$. The isometries in $\mathcal{B}$ with the standard norm are known. Theorem 4.9 gives the isometries in the norm $\|\cdot\|_{Q_{p}}, p>1$.

Theorem 4.7 Let $p>0$ and assume that $C_{\varphi}$ is a bounded operator on $Q_{p}$. Then, there exists $k>0$ such that for all $q \in \mathbb{D},\left\|\alpha_{q} \circ \varphi\right\|_{Q_{p}} \geq k$ if and only if there exists an $\varepsilon>0$ and $r \in(0,1)$ such that for all $q \in \mathbb{D}$, there exists $q^{\prime} \in \mathbb{D}$ such that

$$
\frac{\int_{G_{\varepsilon, q^{\prime}} \cap D(q, r)} N_{q^{\prime}, \varphi}(\zeta) d A(\zeta)}{|D(q, r)|} \asymp 1
$$

Theorem 4.8 Let $p>0$ and assume that $C_{\varphi}$ is a bounded operator on $Q_{p}$. The composition operator $C_{\varphi}$ is closed range on $Q_{p}$ if and only if there exists a constant $k>0$ such that $\left\|\alpha_{q} \circ \varphi\right\|_{Q_{p}} \geq k$, for all $q \in \mathbb{D}$.

Theorem 4.9 Let $p>0$, assume that $C_{\varphi}$ is a bounded operator on $Q_{p}$. The following conditions are equivalent:

(a) There exists $k \in(0,1]$ such that for every $w \in \mathbb{D},\left\|\alpha_{w} \circ \varphi\right\|_{Q_{p}} \geq k$.

(b) There exists $k \in(0,1]$ such that for every $w \in \mathbb{D}$ there exists $w^{\prime} \in \mathbb{D}$ with 
$\left|\alpha_{w}\left(w^{\prime}\right)\right|^{2} \leq 1-k^{2}$, there exists a sequence $\left(q_{n}\right)$ in $\mathbb{D}$ such that $\varphi\left(q_{n}\right) \rightarrow w^{\prime}$ and

$$
\lim _{n \rightarrow \infty}\left\|\varphi_{q_{n}}\right\|_{D_{p}} \geq k
$$

where for all natural numbers $n, \varphi_{q_{n}}=\alpha_{\varphi\left(q_{n}\right)} \circ \varphi \circ \alpha_{q_{n}}$.

We conclude this chapter by providing the following corollary which summarizes our results on closed range composition operators on $\mathcal{B}$.

Corollary 4.10 Let $\varphi$ be a non-constant analytic self-map of $\mathbb{D}$, and $p>1$. Then, the following are equivalent

(a) The composition operator $C_{\varphi}$ is closed range on $\mathcal{B}$.

(b) For all $q \in \mathbb{D},\left\|\alpha_{q} \circ \varphi\right\|_{\mathcal{B}} \asymp 1$.

(c) There exists an $\epsilon>0$ and $r \in(0,1)$ such that for all $q \in \mathbb{D}$, there exists $q^{\prime} \in \mathbb{D}$ such that

$$
\frac{\int_{G_{\varepsilon, q^{\prime}} \cap D(q, r)} N_{q^{\prime}, \varphi}(\zeta) d A(\zeta)}{|D(q, r)|} \asymp 1 .
$$

(d) There exists $k \in(0,1]$ such that for every $w \in \mathbb{D}$ there exists $w^{\prime} \in \mathbb{D}$ with $\left|\alpha_{w}\left(w^{\prime}\right)\right|^{2} \leq 1-k^{2}$, there exists a sequence $\left(q_{n}\right)$ in $\mathbb{D}$ such that $\varphi\left(q_{n}\right) \rightarrow w^{\prime}$ and

$$
\lim _{n \rightarrow \infty}\left\|\varphi_{q_{n}}\right\|_{D_{p}} \geq k
$$

where for all natural numbers $n, \varphi_{q_{n}}=\alpha_{\varphi\left(q_{n}\right)} \circ \varphi \circ \alpha_{q_{n}}$. 


\section{The Bloch space, $B M O A$ and $V M O A$}

Let $\mathbb{D}$ be the open unit disk $\{z \in \mathbb{C}:|z|<1\}$ in the complex plane $\mathbb{C}$ and let $\mathbb{T}$ be the unit circle. A Möbius transformation is a one-to-one analytic function that maps $\mathbb{D}$ onto itself. It has the form $e^{i \theta} \alpha_{q}(z)$ where

$$
\alpha_{q}(z)=\frac{q-z}{1-\bar{q} z}
$$

for $q \in \mathbb{D}$ and $z \in \mathbb{D}$. The set of all Möbius transformations is denoted by $A u t(\mathbb{D})$. Note that $\alpha_{q}$ exchanges the points 0 and $q$ and its inverse under composition is itself. Moreover,

$$
\left|\alpha_{q}^{\prime}(z)\right|=\frac{1-|q|^{2}}{|1-\bar{q} z|^{2}}
$$

and

$$
1-\left|\alpha_{q}(z)\right|^{2}=\frac{\left(1-|q|^{2}\right)\left(1-|z|^{2}\right)}{|1-\bar{q} z|^{2}}=\left(1-|z|^{2}\right)\left|\alpha_{q}^{\prime}(z)\right|
$$

for $q, z \in \mathbb{D}$.

For each $p, q \in \mathbb{D}$

$$
\begin{aligned}
\alpha_{q} \circ \alpha_{p}(z) & =\alpha_{q}\left(\frac{p-z}{1-\bar{p} z}\right) \\
& =\frac{q-\frac{p-z}{1-\bar{p} z}}{1-\bar{q} \frac{p-z}{1-\bar{p} z}} \\
& =\frac{p-q-z(1-q \bar{p})}{-(1-\bar{q} p)+z(\bar{p}-\bar{q})} \\
& =-\frac{1-q \bar{p}}{1-\bar{q} p} \frac{\alpha_{p}(q)-z}{1-\bar{\alpha}_{p}(q) z}
\end{aligned}
$$

Let $b=\alpha_{p}(q)$ and $\rho=-\frac{1-q \bar{p}}{1-\bar{q} p}$. Then, $|\rho|=1$ and we obtain

$$
\alpha_{q} \circ \alpha_{p}=\rho \alpha_{\alpha_{p}(q)} .
$$


The pseudohyperbolic distance $\rho$ between two points $q, z \in \mathbb{D}$ is defined by

$$
\rho(q, z)=\left|\alpha_{q}(z)\right|=\left|\frac{q-z}{1-\bar{q} z}\right|
$$

is invariant under Möbius transformations, that is, for all $z, q \in \mathbb{D}$ and $\varphi \in A u t(\mathbb{D})$.

$$
\rho(\varphi(q), \varphi(z))=\rho(q, z)
$$

and satisfies the following version of triangle inequality, see [12, Lemma 1.4]. Given $z, q, w \in \mathbb{D}$,

$$
\frac{\rho(q, w)-\rho(w, z)}{1-\rho(q, w) \rho(w, z)} \leq \rho(q, z) \leq \frac{\rho(q, w)+\rho(w, z)}{1+\rho(q, w) \rho(w, z)}
$$

Let $A$ denote area measure on $\mathbb{D}$ normalized by the condition $A(\mathbb{D})=1$, for each $H \subseteq \mathbb{D}$, abbreviate $A(H)$ with $|H|$. We denote the euclidean disk centered at $q$ with radius $r \in(0,1)$ with $\mathbb{D}(q, r)$. The pseudohyperbolic disk $D(q, r)$ centered at $q$ with radius $r \in(0,1)$ is

$$
D(q, r)=\{z: \rho(z, q)<r\}
$$

If $r \in(0,1)$ is fixed and $z \in D(q, r)$ then by [37, Proposition 4.5]

$$
A(D(q, r)) \asymp\left(1-|z|^{2}\right)^{2} \asymp|D(q, r)| \asymp\left(1-|q|^{2}\right)^{2},
$$

and

$$
|1-\bar{q} z| \asymp 1-|z|^{2} \asymp 1-|q|^{2} .
$$


Definition 2.1. The Bloch space $\mathcal{B}$ of $\mathbb{D}$ is the space of analytic functions $f$ on $\mathbb{D}$ such that

$$
\|f\|_{\mathcal{B}}=\sup _{z \in \mathbb{D}}\left(1-|z|^{2}\right)\left|f^{\prime}(z)\right|<\infty .
$$

The Bloch space is a Banach space with norm $\|f\|_{B}=|f(0)|+\|f\|_{\mathcal{B}}$. It is invariant under Möbius transformations, that is, if $f \in \mathcal{B}$ then $f \circ \varphi \in \mathcal{B}$, for all Möbius transformation $\varphi$. In fact, by (2) and for all $q, z \in \mathbb{D}$

$$
\begin{aligned}
\left\|f \circ \alpha_{q}\right\|_{\mathcal{B}} & =\sup _{z \in \mathbb{D}}\left(1-|z|^{2}\right)\left|\left(f \circ \alpha_{q}\right)^{\prime}(z)\right| \\
& =\sup _{z \in \mathbb{D}}\left(1-|z|^{2}\right)\left|f^{\prime}\left(\alpha_{q}(z)\right)\right|\left|\alpha_{q}^{\prime}(z)\right| \\
& =\sup _{z \in \mathbb{D}}\left(1-\left|\alpha_{q}(z)\right|^{2}\right)|| f^{\prime}\left(\alpha_{q}(z)\right) \mid \\
& =\sup _{z \in \mathbb{D}}\left|\left(1-|\zeta|^{2}\right)\right| f^{\prime}(\zeta) \mid \\
& =\|f\|_{\mathcal{B}} .
\end{aligned}
$$

The polynomials are not dense in the Bloch space. The closure of the polynomials in the Bloch norm is called the little Bloch space, denoted by $\mathcal{B}_{0}$. It is well known, see for example [37, page 84].

$$
f \in \mathcal{B}_{0} \Leftrightarrow \lim _{|z| \rightarrow 1}\left(1-|z|^{2}\right)\left|f^{\prime}(z)\right|=0 .
$$

Moreover, as Rubel and Timoney showed in [25] the Bloch space is the largest Möbius invariant Banach space that has a non-zero linear functional that extends to a continuous linear functional on $H(\mathbb{D})$, the space of all analytic functions on $\mathbb{D}$.

Definition 2.2. The Bergman space $A^{2}$ is the Hilbert space of analytic functions $f$ on $\mathbb{D}$ that are square-integrable with respect to normalized area measure that is,

$$
\|f\|_{A^{2}}^{2}=\int_{\mathbb{D}}|f(z)|^{2} d A(z)<\infty .
$$


An equivalent norm on $A^{2}$, see for example [37, Theorem 4.28] is given by

$$
\|f\|_{A^{2}}^{2} \asymp|f(0)|^{2}+\int_{\mathbb{D}}\left|f^{\prime}(z)\right|^{2}\left(1-|z|^{2}\right)^{2} d A(z) .
$$

It is well known that the Bloch space can be regarded as the area version of $B M O A$. In particular, by $[1$, Theorem 5.5] and for all $f \in \mathcal{B}$,

$$
\|f\|_{\mathcal{B}} \asymp \sup _{q \in \mathbb{D}}\left\|f \circ \alpha_{q}-f(q)\right\|_{A^{2}}
$$

and by [37, Theorem 4.28],

$$
\|f\|_{\mathcal{B}}^{2} \asymp \sup _{q \in \mathbb{D}} \int_{\mathbb{D}}\left|f^{\prime}(z)\right|^{2}\left(1-\left|\alpha_{q}(z)\right|^{2}\right)^{2} d A(z) .
$$

Definition 2.3. The Dirichlet space $\mathcal{D}$ is the space of analytic functions on $\mathbb{D}$ such that

$$
\|f\|_{\mathcal{D}}=|f(0)|^{2}+\int_{\mathbb{D}}\left|f^{\prime}(z)\right|^{2} d A(z)<\infty .
$$

Next, we give the definition of analytic $B M O$, namely $B M O A$ space which is a Möbius invariant version of the Hardy Space $H^{2}$.

Definition 2.4. An analytic function $f \in H(\mathbb{D})$ belongs to $B M O A$ if

$$
\|f\|_{G}=\sup _{q \in \mathbb{D}}\left\|\mid f \circ \alpha_{q}-f(q)\right\|_{H^{2}}<\infty
$$

We note that $\|f\|_{G}$ defines a seminorm and $|f(0)|+\|f\|_{G}$ is a norm in BMOA that makes it a Banach space.

Notation $S(h, \theta)=\left\{z \in \mathbb{D}:\left|z-e^{i \theta}\right|<h\right\}$, where $\theta \in[0,2 \pi), h \in(0,1)$.

The notion of $B M O A$ first arose in the context of mean oscillation of a function over cubes with edges parallel to the coordinate axes or equivalently over sets of the form 
$S(h, \theta)([28$, pages, 36-39]). That is,

$$
\|f\|_{*}^{2} \asymp \sup _{h \in(0,1), \theta \in[0,2 \pi)} \frac{1}{h} \int_{S(h, \theta)}\left|f^{\prime}(z)\right|^{2}\left(1-|z|^{2}\right) d A(z) .
$$

Given $h>0$ and $\theta \in \mathbb{R}$, let

$$
Q=\left\{r e^{i \theta}: \theta_{0}<\theta<\theta_{0}+h, 1-h<r<1\right\}
$$

A positive measure $\mu$ in $\mathbb{D}$ is a Carleson measure for the Hardy space if there exists a constant $k>0$ such that $\mu(Q) \leq k h$, for all $h>0$. It is known, see for example $[12$, Lemma 3.3], that $\mu$ is a Carleson measure for the Hardy space if and only if

$$
\sup _{q \in \mathbb{D}} \int_{\mathbb{D}} \frac{1-|q|^{2}}{|1-\bar{q} z|^{2}} d \mu(z)<\infty
$$

Then $f \in B M O A$ if and only if $\left|f^{\prime}(z)\right|^{2}\left(1-|z|^{2}\right) d A(z)$ is a Carleson measure for the Hardy space.

Theorem 2.1. [31, page 178] (The Littlewood-Paley Identity) If $f$ is an analytic on $\mathbb{D}$, then

$$
\|f\|_{H^{2}}^{2}=|f(0)|^{2}+2 \int_{\mathbb{D}}\left|f^{\prime}(z)\right|^{2} \log \frac{1}{|z|} d A(z) .
$$

The following allows us to obtain a useful variation of the Littlewood-Paley identity, see [31, page 178] and [12, Lemma 3.2$]$.

Proposition 2.2. For every analytic function $g$ on $\mathbb{D}$,

$$
\int_{\mathbb{D}}|g(z)| \log \frac{1}{|z|} d A(z) \asymp \int_{\mathbb{D}}|g(z)|\left(1-|z|^{2}\right) d A(z) .
$$


Proof. We know that for all $z \in \mathbb{D} \backslash\{0\}$

$$
1-|z|^{2} \leq 2 \log \frac{1}{|z|}
$$

and $|z|>1 / 4$ implies that there exists a constant $c_{1}>0$ such that

$$
\log \frac{1}{|z|} \leq c_{1}\left(1-|z|^{2}\right)
$$

Let $g$ be an analytic function on $\mathbb{D}$. Then by (13)

$$
\int_{\mathbb{D}}|g(z)|\left(1-|z|^{2}\right) d A(z) \leq 2 \int_{\mathbb{D}}|g(z)| \log \frac{1}{|z|} d A(z),
$$

and $|g|$ is subharmonic. Therefore, if $|z| \leq 1 / 4$ then

$$
\begin{aligned}
|g(z)| & \leq 16 \int_{D(z, 1 / 4)}|g(\zeta)| d A(\zeta) \\
& =16 \int_{D(z, 1 / 4)} \frac{|g(\zeta)|\left(1-|\zeta|^{2}\right)}{1-|\zeta|^{2}} d A(\zeta) .
\end{aligned}
$$

Notice that if $\zeta \in D(z, 1 / 4)$, then $|\zeta| \leq 1 / 2$ and

$$
|g(z)| \leq 22 \int_{\mathbb{D}}|g(\zeta)|\left(1-|\zeta|^{2}\right) d A(\zeta) .
$$

By (14)

$$
\int_{\mathbb{D} \backslash D(0,1 / 4)}|g(z)| \log \frac{1}{|z|} d A(z) \leq c_{1} \int_{\mathbb{D}}|g(z)|\left(1-|z|^{2}\right) d A(z)
$$


and by (16)

$$
\begin{aligned}
\int_{D(0,1 / 4)}|g(z)| \log \frac{1}{|z|} d A(z) & \leq 22 \int_{D(0,1 / 4)} \log \frac{1}{|z|} \int_{\mathbb{D}}|g(\zeta)|\left(1-|\zeta|^{2}\right) d A(z) d A(\zeta) \\
& =22\left(\int_{D(0,1 / 4)} \log \frac{1}{|z|} d z\right) \int_{\mathbb{D}}|g(\zeta)|\left(1-|\zeta|^{2}\right) d A(\zeta) .
\end{aligned}
$$

Notice that

$$
\begin{aligned}
\int_{D(0,1 / 4)} \log \frac{1}{|z|} d A(z) & =\int_{0}^{1 / 4} \int_{0}^{2 \pi} \log \frac{1}{r} r d r \frac{d \theta}{\pi} \\
& =2 \int_{0}^{1 / 4} r \log \frac{1}{r} d r \\
& \asymp .1178934,
\end{aligned}
$$

and there exists $c>0$ such that

$$
\int_{D(0,1 / 4)}|g(z)| \log \frac{1}{|z|} d A(z) \leq c \int_{\mathbb{D}}|g(\zeta)|\left(1-|\zeta|^{2}\right) d A(\zeta) .
$$

Therefore by (15), (17), and (18) we arrive at the conclusion.

By the Littlewood-Paley identity

$$
\|f\|_{G}^{2}=\sup _{q \in \mathbb{D}} \int_{\mathbb{D}}\left|\left(f \circ \alpha_{q}\right)^{\prime}(z)\right|^{2} \log \frac{1}{|z|} d A(z)
$$

and by Proposition 2.2, $\|f\|_{G} \asymp\|f\|_{*}$ where

$$
\|f\|_{*}^{2}=\sup _{q \in \mathbb{D}} \int_{\mathbb{D}}\left|\left(f \circ \alpha_{q}\right)^{\prime}(z)\right|^{2}\left(1-|z|^{2}\right) d A(z) .
$$


Note that by (19) and by making change of variables $\alpha_{q}(z)=w$ we obtain

$$
\begin{aligned}
\|f\|_{*}^{2} & =\sup _{q \in \mathbb{D}} \int_{\mathbb{D}}\left|\left(f^{\prime}\left(\alpha_{q}(z)\right)\right)\right|^{2}\left|\alpha_{q}^{\prime}(z)\right|^{2}\left(1-|z|^{2}\right) d A(z) \\
& =\sup _{q \in \mathbb{D}} \int_{\mathbb{D}}\left|f^{\prime}(w)\right|^{2}\left(1-\left|\alpha_{q}(w)\right|^{2}\right) d A(w) .
\end{aligned}
$$

There are a number of equivalent norms in $B M O A$. We will use

$$
\|f\|_{B M O A}=|f(0)|+\|f\|_{*} .
$$

It is easy to see that $B M O A$ is a Möbius invariant Banach space. Let $f \in B M O A, p \in \mathbb{D}$. Then by (20)

$$
\begin{aligned}
\left\|f \circ \alpha_{p}\right\|_{*}^{2} & =\sup _{q \in \mathbb{D}} \int_{\mathbb{D}}\left|\left(f \circ \alpha_{p}\right)^{\prime}(z)\right|^{2}\left(1-\left|\alpha_{q}(z)\right|^{2}\right) d A(z) \\
& =\sup _{q \in \mathbb{D}} \int_{\mathbb{D}} \mid\left(\left.f^{\prime}\left(\alpha_{p}(z)\right)\right|^{2}\left|\alpha_{p}^{\prime}(z)\right|^{2}\left(1-\left|\alpha_{q}(z)\right|^{2}\right) d A(z)\right. \\
& =\sup _{q \in \mathbb{D}} \int_{\mathbb{D}}\left|f^{\prime}(\zeta)\right|^{2}\left(1-\left|\alpha_{q}\left(\alpha_{p}(\zeta)\right)\right|^{2}\right) d A(\zeta) .
\end{aligned}
$$

For each $q \in \mathbb{D}$ and by (3), let $\alpha_{q} \circ \alpha_{p}=e^{i \theta} \alpha_{Q}$ for some $Q \in \mathbb{D}, \theta \in \mathbb{R}$; then

$$
\begin{aligned}
\left\|f \circ \alpha_{p}\right\|_{*}^{2} & =\sup _{Q \in \mathbb{D}} \int_{\mathbb{D}}\left|f^{\prime}(w)\right|^{2}\left(1-\left|\alpha_{Q}(w)\right|^{2}\right) d A(w) \\
& =\|f\|_{*}^{2} .
\end{aligned}
$$


For each $q \in \mathbb{D}$ and since $B M O A$ is a Möbius invariant Banach space, $\left\|\alpha_{q}\right\|_{*}=\|z\|_{*}$,

$$
\begin{aligned}
\left\|\alpha_{q}\right\|_{*} & \asymp\|z\|_{G} \\
& =\sup _{q \in \mathbb{D}}\left\|\alpha_{q}-q\right\|_{H^{2}} \\
& =\sup _{q \in \mathbb{D}}\left\|\frac{z\left(1-|q|^{2}\right)}{1-\bar{q} z}\right\|_{H^{2}} \\
& =\sup _{q \in \mathbb{D}}\left(1-|q|^{2}\right)\left\|\frac{z}{1-\bar{q} z}\right\|_{H^{2}} \\
& =\sup _{q \in \mathbb{D}}\left(1-|q|^{2}\right)\left\|z \sum_{n=0}^{\infty}(\bar{q} z)^{n}\right\|_{H^{2}} \\
& =\sup _{q \in \mathbb{D}}\left(1-|q|^{2}\right)|| \sum_{n=1}^{\infty}(\bar{q})^{n-1} z^{n}|| \\
& =\sup _{q \in \mathbb{D} \backslash\{0\}}\left(1-|q|^{2}\right) \frac{1}{|q|}\left(\sum_{n=1}^{\infty}|q|^{2 n}\right)^{\frac{1}{2}} \\
& =\sup _{q \in \mathbb{D}}\left(1-|q|^{2}\right) \frac{1}{|q|}\left(\frac{1}{1-|q|^{2}}-1\right)^{\frac{1}{2}} \\
& =1 .
\end{aligned}
$$

One of the many similarities between the Bloch space and $B M O A$ is that polynomials are not dense in either space. The closure of the polynomials in the BMOA norm is $V M O A$, the space of analytic functions with vanishing mean oscillation. The space VMOA can be characterized as all those analytic functions $f$ on $\mathbb{D}$ such that

$$
\lim _{|q| \rightarrow 1} \int_{\mathbb{D}}\left|f^{\prime}(w)\right|^{2}\left(1-\left|\alpha_{q}(w)\right|^{2} d A(w)=0\right.
$$

(the "little-oh" version of (20)).

We can see that $B M O A$ is a subspace of the Bloch space as follows:

$$
\left|f^{\prime}(0)\right| \leq\|f\|_{H^{2}}
$$


for any $f$ analytic on $\mathbb{D}$; therefore there exists $c>0$ such that for each $q \in \mathbb{D}$

$$
\begin{aligned}
\left|f^{\prime}(q)\right|\left(1-|q|^{2}\right) & =\left|\left(f \circ \alpha_{q}-f(q)\right)^{\prime}(0)\right| \\
& \leq\left\|f \circ \alpha_{q}-f(q)\right\|_{H^{2}} \\
& \leq c\|f\|_{*} .
\end{aligned}
$$

Hence, $\|f\|_{\mathcal{B}} \leq c\|f\|_{*}$ for all $f \in B M O A$ and $B M O A \subset \mathcal{B}$ and the inclusion map $i: B M O A \rightarrow \mathcal{B}$ is continuous.

Below we establish the growth of $B M O A$ functions. Let $f \in B M O A$. Then $f$ is analytic on $\mathbb{D}$ and for all $z \in \mathbb{D}$,

$$
|f(z)-f(0)|=\left|z \int_{0}^{1} f^{\prime}(t z) d t\right| .
$$

Since the inclusion map $i: B M O A \rightarrow \mathcal{B}$ is continuous, there exists $c>0$ such that for all $z \in \mathbb{D}$

$$
\left|f^{\prime}(z)\right|\left(1-|z|^{2}\right) \leq c|| f \|_{*}
$$

and

$$
\begin{aligned}
|f(z)-f(0)| & \leq|z| \int_{0}^{1}\left|f^{\prime}(t z)\right| d t \\
& \leq c|z|\|f\|_{*} \int_{0}^{1} \frac{1}{1-t^{2}|z|^{2}} d t \\
& =\frac{c}{2}\|f\|_{*} \log \frac{1+|z|}{1-|z|} .
\end{aligned}
$$

Therefore,

$$
|f(z)| \leq|f(0)|+\left.\frac{c}{2}|| f\right|_{*} \log \frac{1+|z|}{1-|z|} .
$$


Finally we note that

$$
\mathcal{D} \subset V M O A \subset B M O A \subset \mathcal{B} \subset \mathrm{A}^{2}
$$

Let $H^{\infty}$ denote the set of all functions in $H(\mathbb{D})$ that are bounded. Given $f \in H^{\infty}$ let

$$
\|f\|_{\infty}=\sup _{z \in \mathbb{D}}|f(z)|
$$

By applying Schwarz Lemma we obtain that for all $z \in \mathbb{D},\left|f^{\prime}(z)\right|\left(1-|z|^{2}\right) \leq\|f\|_{\infty}$, see $[37$, page 101]. By (10) it is now easy to see that $H^{\infty} \subset B M O A$. There are unbounded functions in $B M O A$.

The function $\log (1-z) \in B M O A$. In fact, if $f$ is any analytic, univalent, and zero free function then $\log f \in B M O A$ (This result first appeared in [5] and [10]). Below we give more examples of $B M O A$ functions.

If $\left(a_{n}\right)$ is a bounded sequence then $\sum_{n=1}^{\infty} \frac{1}{n} a_{n} z^{n} \in B M O A$, and if $\sum_{n=1}^{\infty}\left|a_{n}\right|^{2}<\infty$ then $\sum_{n=0}^{\infty} a_{n} z^{\lambda_{n}} \in B M O A$, where $\left(\lambda_{n}\right)$ is a sequence of integers satisfying

$$
\frac{\lambda_{n+1}}{\lambda_{n}} \geq \lambda>1
$$

$\lambda$ is a constant and $n$ is a natural number. 


\section{Bounded and closed range composition operators on $B M O A$}

Given $\varphi$ an analytic self-map of $\mathbb{D}$, the composition operator, $C_{\varphi}$ is defined for all $f \in H(\mathbb{D})$ as follows,

$$
C_{\varphi} f=f \circ \varphi .
$$

It is well known that all composition operators $C_{\varphi}$ are bounded on the Hardy space $H^{2}$ and, if $\varphi(0)=0$, then $C_{\varphi}$ is a contraction.

Theorem 3.1. [31, page 13] Littlewood's Subordination Principle (1925)

Suppose $\varphi$ is an analytic self-map of $\mathbb{D}$, with $\varphi(0)=0$. Then for each $f \in H^{2}$, $C_{\varphi} f \in H^{2}$ and $\left\|C_{\varphi} f\right\|_{H^{2}} \leq\|f\|_{H^{2}}$.

More generally, we have the following.

Theorem 3.2. [31, page 13] Littlewood's Theorem (1925)

Let $\varphi: \mathbb{D} \rightarrow \mathbb{D}$ be an analytic function. Then $C_{\varphi}$ is bounded on $H^{2}(\mathbb{D})$, and

$$
\left\|C_{\varphi} f\right\|_{H^{2}} \leq \sqrt{\frac{1+|\varphi(0)|}{1-|\varphi(0)|}}\|f\|_{H^{2}} .
$$

Definition 3.1. The Nevanlinna counting function of $\varphi$ is

$$
N_{\varphi}(w)=\sum_{\varphi(z)=w} \log \frac{1}{|z|}
$$

if $w \in \varphi(\mathbb{D})$; if $w \notin \varphi(\mathbb{D})$ then we set $N_{\varphi}(w)=0$.

Theorem 3.3. [31, page 187] Littlewood's Inequality If $\varphi$ is an analytic self-map of $\mathbb{D}$, then for each $w \in \mathbb{D} \backslash\{\varphi(0)\}$,

$$
N_{\varphi}(w) \leq \log \left|\frac{1-\bar{w} \varphi(0)}{w-\varphi(0)}\right| .
$$


Definition 3.2. For each $q \in \mathbb{D}$, and if $\zeta \in \varphi(\mathbb{D})$ the BMOA counting function is

$$
N_{q, \varphi}(\zeta)=\sum_{\varphi(z)=\zeta}\left(1-\left|\alpha_{q}(z)\right|^{2}\right) ;
$$

if $\zeta \notin \varphi(\mathbb{D})$ then we set $N_{q, \varphi}(\zeta)=0$.

Counting functions have played an important role in the study of compact, bounded and closed range composition operators. The Nevanlinna counting function for composition operators in BMOA appears with a non-univalent change of variables as it is done in [31, page 186].

For each $f \in B M O A$,

$$
\begin{aligned}
\left\|C_{\varphi} f\right\|_{*}^{2} & =\sup _{q \in \mathbb{D}} \int_{\mathbb{D}}\left|(f \circ \varphi)^{\prime}(z)\right|^{2}\left(1-\left|\alpha_{q}(z)\right|^{2}\right) d A(z) \\
& =\sup _{q \in \mathbb{D}} \int_{\mathbb{D}} \mid\left(\left.f^{\prime}(\varphi(z))\right|^{2}\left|\varphi^{\prime}(z)\right|^{2}\left(1-\left|\alpha_{q}(z)\right|^{2}\right) d A(z) .\right.
\end{aligned}
$$

Then we have

$$
\left\|C_{\varphi} f\right\|_{*}^{2}=\sup _{q \in \mathbb{D}} \int_{\mathbb{D}}\left|f^{\prime}(\zeta)\right|^{2} N_{q, \varphi}(\zeta) d A(\zeta) .
$$

It is well known that $C_{\varphi}$ is always bounded on $B M O A$, see [4, Theorem 12]. Littlewood's Subordination Principle provides a proof, see for example [33]. Smith points out in [32] that Littlewood's inequality provides an alternate proof, which we will describe below for our chosen norm.

Theorem 3.4. Let $\varphi$ be an analytic self-map of $\mathbb{D}$. Then $C_{\varphi}$ is a bounded operator on $B M O A$.

Proof. For all $z \in \mathbb{D} \backslash\{0\}$ we have,

$$
1-|z|^{2} \leq \log \frac{1}{|z|^{2}}
$$


therefore by (26)

$$
\left\|C_{\varphi} f\right\|_{*}^{2} \leq \sup _{q \in \mathbb{D}} \int_{\mathbb{D}}\left|f^{\prime}(\zeta)\right|^{2} \sum_{\varphi(z)=\zeta} \log \frac{1}{\left|\alpha_{q}(z)\right|^{2}} d A(\zeta)
$$

By Proposition 2.2 and Littlewood's Inequality, there exists $C>0$ such that

$$
\begin{aligned}
\left\|C_{\varphi} f\right\|_{*}^{2} & \leq \sup _{q \in \mathbb{D}} \int_{\mathbb{D}}\left|f^{\prime}(\zeta)\right|^{2} \log \frac{1}{\left|\alpha_{\varphi(q)}(\zeta)\right|^{2}} d A(\zeta) \\
& \leq \sup _{q \in \mathbb{D}} \int_{\mathbb{D}}\left|f^{\prime}(\zeta)\right|^{2} \log \frac{1}{\left|\alpha_{q}(\zeta)\right|^{2}} d A(\zeta) \\
& \leq C\|f\|_{*}^{2},
\end{aligned}
$$

and $C_{\varphi}$ is a bounded operator on $B M O A$.

Next, let $X=\mathcal{B}, B M O A$ or $V M O A$ and $Y=B M O A$ or $V M O A$. We show that for $C_{\varphi}$ to be bounded below, we can restrict on subspaces of functions that vanish at the origin. The canonical seminorm in $X$ is denoted by $\|\cdot\|_{s X}$.

Proposition 3.5. Let $X=\mathcal{B}, B M O A$ or $V M O A$ and $Y=B M O A$ or $V M O A$. The composition operator $C_{\varphi}: X \rightarrow Y$ is bounded below if and only if there exists a $\delta>0$ such that $\left\|C_{\varphi} f\right\|_{*} \geq \delta\|f\|_{s X}$, for all $f \in X$.

Proof. First, assume that $C_{\varphi}: X \rightarrow B M O A$ is bounded below. Let $f \in X$ and $g(z)=f(z)-f(\varphi(0))$. Then, $g \in X, g(\varphi(0))=0,\|g\|_{s X}=\|f\|_{s X}$ and $\|g \circ \varphi\|_{*}=\|f \circ \varphi\|_{*}$. Thus, there exists $\delta>0$ such that

$$
\left\|C_{\varphi} f\right\|_{*}=\|f \circ \varphi\|_{*}=\|g \circ \varphi\|_{*}=\|g \circ \varphi\|_{B M O A} \geq \delta\|g\|_{X} \geq \delta\|g\|_{s X}=\delta\|f\|_{s X} .
$$

Conversely, assume that there exists a $\delta>0$ such that $\|f \circ \varphi\|_{*} \geq \delta\|f\|_{s X}$, for all $f \in X$. Then by (24) there exists $C>0$ such that

$$
|f(0)| \leq|f(\varphi(0))|+C|| f \|_{s X} \log \frac{1+|\varphi(0)|}{1-|\varphi(0)|},
$$


which implies that there exists $C>0$ such that

$$
\begin{aligned}
\|f\|_{X} & =|f(0)|+\|f\|_{s X} \\
& \leq|f(\varphi(0))|+C\|f\|_{s X} \log \frac{1+|\varphi(0)|}{1-|\varphi(0)|}+\frac{1}{\delta}\|f \circ \varphi\|_{*} \\
& \leq|f(\varphi(0))|+\frac{c}{\delta}\|f \circ \varphi\|_{*} \log \frac{1+|\varphi(0)|}{1-|\varphi(0)|}+\frac{1}{\delta}\|f \circ \varphi\|_{*} \\
& \leq C\|f \circ \varphi\|_{\text {BMOA }} .
\end{aligned}
$$

Hence, $C_{\varphi}$ is bounded below on $B M O A$.

Definition 3.3. Let $\mu$ be a finite positive Borel measure on $\mathbb{D}$. We say that $\mu$ is a Carleson measure for the Bergman space if there exists constant $c>0$ such that for all $f \in A^{2}$

$$
\int_{\mathbb{D}}|f(z)|^{2} d \mu(z) \leq c \int_{\mathbb{D}}|f(z)|^{2} d A(z)
$$

By [37, Theorem 7.4], given $r \in(0,1), \mu$ is a Carleson measure if and only if there exists $c_{r}>0$ such that for all $w \in \mathbb{D}$,

$$
\mu(D(w, r)) \leq c_{r}|D(w, r)|
$$

The Berezin symbol of $\mu$ is

$$
\tilde{\mu}(q)=\int_{\mathbb{D}}\left|\alpha_{q}^{\prime}(z)\right|^{2} d \mu(z), \quad q \in \mathbb{D}
$$

It is well known that $\mu$ is a Carleson measure for the Bergman space if and only if $\tilde{\mu}$, is a bounded function on $\mathbb{D}$, that is,

$$
\sup _{q \in \mathbb{D}} \int_{\mathbb{D}}\left|\alpha_{q}^{\prime}(z)\right|^{2} d \mu(z) \lesssim 1
$$

see for example [37, Theorem 7.5]. The proof of (30) can be used to show that $\mu_{q^{\prime}}, q^{\prime} \in \mathbb{D}$, 
is a collection of uniformly Carleson measures for the Bergman space if and only if

$$
\sup _{w, q^{\prime} \in \mathbb{D}} \int_{\mathbb{D}}\left|\alpha_{w}^{\prime}(z)\right|^{2} d \mu(z) \lesssim 1
$$

For any symbol $\varphi$ and by Theorem 3.4, $C_{\varphi}$ is a bounded operator on $B M O A$. Then for all $q \in \mathbb{D},\left\|C_{\varphi} \alpha_{q}\right\|_{*} \lesssim 1$ and by $(26)$

$$
\sup _{q, q^{\prime} \in \mathbb{D}} \int_{\mathbb{D}}\left|\alpha_{q}^{\prime}(\zeta)\right|^{2} N_{q^{\prime}, \varphi}(\zeta) d A(\zeta) \lesssim 1
$$

By (31) and for all $q^{\prime} \in \mathbb{D}, N_{q^{\prime}, \varphi}(\zeta) d A(\zeta)$ is a collection of uniformly Carleson measures for the Bergman space.

Definition 3.4. Let $\mu$ be a finite positive Carleson measure on $\mathbb{D}$. We say that $\mu$ satisfies the reverse Carleson condition if there exists $r \in(0,1)$ such that for all $w \in \mathbb{D}$,

$$
|D(w, r)| \asymp \mu(D(w, r)) .
$$

It is shown in [16, Theorem 4.1] that $\mu$ satisfies the reverse Carleson condition if and only if

$$
\sup _{q \in \mathbb{D}} \int_{\mathbb{D}}\left|\alpha_{q}^{\prime}(z)\right|^{2} d \mu \asymp 1
$$

A set $H \subset \mathbb{D}$ satisfies the reverse Carleson condition if the Carleson measure $\chi_{H}(z) d A(z)$ satisfies the reverse Carleson condition; Luecking showed in [20] that this is equivalent to

$$
\int_{\mathbb{D}}|f(z)|^{2} d A(z) \leq C \int_{H}|f(z)|^{2} d A(z),
$$

for all $f \in A^{2}$. It is also equivalent to

$$
\int_{\mathbb{D}}\left|f^{\prime}(z)\right|^{2}\left(1-|z|^{2}\right) d A(z) \leq c \int_{H}\left|f^{\prime}(z)\right|^{2}\left(1-|z|^{2}\right) d A(z),
$$


for all $f \in H^{2}$.

Definition 3.5. We say that $H \subseteq \mathbb{D}$ is a sampling set for $B M O A$ if for all $f \in B M O A$

$$
\sup _{q \in \mathbb{D}} \int_{H}\left|f^{\prime}(z)\right|^{2}\left(1-\left|\alpha_{q}(z)\right|^{2}\right) d A(z) \asymp\|f\|_{*}^{2} .
$$

For each $\varepsilon>0$ and $q \in \mathbb{D}$ let $G_{\varepsilon, q}$ be

$$
G_{\varepsilon, q}=\left\{\zeta: N_{q, \varphi}(\zeta)>\varepsilon\left(1-\left|\alpha_{q}(\zeta)\right|^{2}\right)\right\}
$$

and let $G_{\varepsilon}$ be

$$
G_{\varepsilon}=\cup_{q \in \mathbb{D}} G_{\varepsilon, q}
$$

Theorem 3.6. Let $\varphi$ be a non-constant analytic self map of $\mathbb{D}$. If $C_{\varphi}$ is closed range on $B M O A$ then there exists $\varepsilon>0$ such that $G_{\varepsilon}$ is a sampling set for BMOA. Moreover, if $\cap_{q \in \mathbb{D}} G_{\varepsilon, q}$ is a sampling set for $B M O A$, then $C_{\varphi}$ is closed range on BMOA.

Proof. First, suppose that $C_{\varphi}$ is closed range on $B M O A$. If the conclusion fails, then for every $\varepsilon>0, G_{\varepsilon}$ is not a sampling set for $B M O A$ and we can find a sequence $\left(f_{n}\right)$ in $B M O A$ with $f_{n}(0)=0$ and $\left\|f_{n}\right\|_{B M O A}=1$ for all $n$ such that

$$
\lim _{n \rightarrow \infty} \sup _{q \in \mathbb{D}} \int_{G_{\frac{1}{n}}}\left|f_{n}^{\prime}(z)\right|^{2}\left(1-\left|\alpha_{q}(z)\right|^{2}\right) d A(z)=0
$$

For each $n$ we have,

$$
\begin{aligned}
\left\|f_{n} \circ \varphi\right\|_{*}^{2} & =2 \sup _{q \in \mathbb{D}} \int_{\mathbb{D}}\left|f_{n}^{\prime}(\zeta)\right|^{2} N_{q, \varphi}(\zeta) d A(\zeta) \\
& \leq I_{1}+I_{2}
\end{aligned}
$$


where

$$
I_{1}=2 \sup _{q \in \mathbb{D}} \int_{G_{\frac{1}{n}}}\left|f_{n}^{\prime}(\zeta)\right|^{2} N_{q, \varphi}(\zeta) d A(\zeta)
$$

and

$$
I_{2}=2 \sup _{q \in \mathbb{D}} \int_{\mathbb{D} \backslash G_{\frac{1}{n}}}\left|f_{n}^{\prime}(\zeta)\right|^{2} N_{q, \varphi}(\zeta) d A(\zeta)
$$

By Littlewood's inequality, there exists $C>0$ such that for all $\zeta \neq \varphi(q)$,

$$
\sum_{\varphi(z)=\zeta} \log \frac{1}{\left|\alpha_{q}(z)\right|^{2}} \leq C \log \frac{1}{\left|\alpha_{\varphi(q)}(\zeta)\right|^{2}}
$$

For all $n$,

$$
\begin{aligned}
I_{1} & \leq C \sup _{q \in \mathbb{D}} \int_{G_{\frac{1}{n}}}\left|f_{n}^{\prime}(\zeta)\right|^{2} \sum_{\varphi(z)=\zeta} \log \frac{1}{\left|\alpha_{q}(z)\right|^{2}} d A(\zeta) \\
& \leq C \sup _{q \in \mathbb{D}} \int_{G_{\frac{1}{n}}}\left|f_{n}^{\prime}(\zeta)\right|^{2} \log \frac{1}{\left|\alpha_{\varphi(q)}(\zeta)\right|^{2}} d A(\zeta)
\end{aligned}
$$

Note that for any $q \in \mathbb{D}$ and for any natural number $n$,

$$
\int_{G_{\frac{1}{n}}}\left|f_{n}^{\prime}(\zeta)\right|^{2} \log \frac{1}{\left|\alpha_{q}(\zeta)\right|^{2}} d A(\zeta)=A-B
$$

where

$$
A=\int_{\mathbb{D}}\left|f_{n}^{\prime}(\zeta)\right|^{2} \log \frac{1}{\left|\alpha_{q}(\zeta)\right|^{2}} d A(\zeta)
$$

and

$$
B=\int_{\mathbb{D} \backslash G_{\frac{1}{n}}}\left|f_{n}^{\prime}(\zeta)\right|^{2} \log \frac{1}{\left|\alpha_{q}(\zeta)\right|^{2}} d A(\zeta)
$$


Then by Proposition 2.2,

$$
A \leq C \int_{\mathbb{D}}\left|f_{n}^{\prime}(\zeta)\right|^{2}\left(1-\left|\alpha_{q}(\zeta)\right|^{2}\right) d A(\zeta)
$$

and by (13),

$$
B \geq \frac{1}{2} \int_{\mathbb{D} \backslash G_{\frac{1}{n}}}\left|f_{n}^{\prime}(\zeta)\right|^{2}\left(1-\left|\alpha_{q}(\zeta)\right|^{2}\right) d A(\zeta)
$$

We conclude that

$$
I_{1} \leq C \sup _{q \in \mathbb{D}} \int_{G_{\frac{1}{n}}}\left|f_{n}^{\prime}(\zeta)\right|^{2}\left(1-\left|\alpha_{q}(\zeta)\right|^{2}\right) d A(\zeta) .
$$

Next, for $n$ a fixed natural number, $\zeta \notin G_{1 / n}$, and $q \in \mathbb{D}$,

$$
N_{q, \varphi}(\zeta) \leq \frac{1}{n}\left(1-\left|\alpha_{q}(\zeta)\right|^{2}\right),
$$

and

$$
\begin{aligned}
I_{2} & \leq \frac{1}{n} \sup _{q \in \mathbb{D}} \int_{\mathbb{D} \backslash G_{\frac{1}{n}}}\left|f_{n}^{\prime}(\zeta)\right|^{2}\left(1-\left|\alpha_{q}(\zeta)\right|^{2}\right) d A(\zeta) \\
& \leq \frac{1}{n} \sup _{q \in \mathbb{D}} \int_{\mathbb{D}}\left|f_{n}^{\prime}(\zeta)\right|^{2}\left(1-\left|\alpha_{q}(\zeta)\right|^{2}\right) d A(\zeta) \\
& \leq \frac{1}{n}\left\|f_{n}\right\|_{*}^{2} \\
& =\frac{1}{n}
\end{aligned}
$$

By (34), (35), (39) and (40), $\left\|f_{n} \circ \varphi\right\|_{*} \rightarrow 0$, as $n \rightarrow \infty$ and $C_{\varphi}$ is not closed range on $B M O A$ which contradicts our hypothesis and the conclusion holds.

Conversely, assume that there exists $\varepsilon>0$ such that $\cap_{q \in \mathbb{D}} G_{\varepsilon, p}$ is a sampling set for 
$B M O A$. Let $f \in B M O A$. Then, for all $q \in \mathbb{D}$

$$
\begin{aligned}
\|f \circ \varphi\|_{*}^{2} & \geq \int_{G_{\varepsilon, q}}\left|f^{\prime}(\zeta)\right|^{2} N_{q, \varphi}(\zeta) d A(\zeta) \\
& \geq \varepsilon \int_{G_{\varepsilon, q}}\left|f^{\prime}(\zeta)\right|^{2}\left(1-\left|\alpha_{q}(\zeta)\right|^{2}\right) d A(\zeta) \\
& \geq \int_{\cap_{q \in \mathbb{D}} G_{\varepsilon, q}}\left|f^{\prime}(\zeta)\right|^{2}\left(1-\left|\alpha_{q}(\zeta)\right|^{2}\right) d A(\zeta) .
\end{aligned}
$$

Since $G_{\varepsilon}$ is a sampling set for $B M O A$ we conclude that $C_{\varphi}$ is closed range on $B M O A$.

The following is a corollary of the proof of [16, Proposition 4.1].

Theorem 3.7. Let $\mu_{q}, q \in \mathbb{D}$ be a collection of positive uniformly Carleson measures, let $\alpha, \beta$ be such that $\alpha+\beta=2$. Then

$$
\lim _{r \rightarrow 1} \sup _{q, w \in \mathbb{D}} \int_{\mathbb{D} \backslash D(w, r)} \frac{\left(1-|w|^{2}\right)^{\alpha}\left(1-|\zeta|^{2}\right)^{\beta}}{|1-\bar{w} \zeta|^{4}} d \mu_{q}(\zeta)=0 .
$$

For each $\varepsilon>0$ and $q, q^{\prime} \in \mathbb{D}$ let $G_{\varepsilon, q^{\prime}, q}$ be

$$
G_{\varepsilon, q^{\prime}, q}=\left\{\zeta: N_{q^{\prime}, \varphi}(\zeta) \geq \varepsilon\left(1-\left|\alpha_{q}(\zeta)\right|^{2}\right)\right\}
$$

Theorem 3.8. Let $\varphi$ be an analytic self-map of $\mathbb{D}$. Then, there exists $k>0$ such that for all $q \in \mathbb{D},\left\|\alpha_{q} \circ \varphi\right\|_{*} \geq k$ if and only if there exists an $\varepsilon>0$ and $r \in(0,1)$ such that for all $q \in \mathbb{D}$, there exists $q^{\prime} \in \mathbb{D}$ such that

$$
\frac{\left|G_{\varepsilon, q^{\prime}, q} \cap D(q, r)\right|}{|D(q, r)|} \asymp 1
$$

Moreover, if the above holds then $G_{\varepsilon}=\cup_{q \in \mathbb{D}} G_{\varepsilon, q^{\prime}, q}$ satisfies the reverse Carleson condition.

Proof. First, assume that there exists $k>0$ such that for all $q$ in $\mathbb{D},\left\|\alpha_{q} \circ \varphi\right\|_{*} \geq k$. Then, for each $q \in \mathbb{D}$ there exists a $q^{\prime} \in \mathbb{D}$ such that 


$$
\int_{\mathbb{D}}\left|\alpha_{q}^{\prime}(\zeta)\right|^{2} N_{q^{\prime}, \varphi}(\zeta) d A(\zeta) \geq \frac{k}{2}
$$

By Theorem 3.7 and since for all $q^{\prime} \in \mathbb{D}, N_{q^{\prime}, \varphi}(\zeta) d A(\zeta)$ is a collection of uniformly Carleson measures for the Bergman space,

$$
\lim _{r \rightarrow 1} \sup _{q, q^{\prime} \in \mathbb{D}} \int_{\mathbb{D} \backslash D(q, r)}\left|\alpha_{q}^{\prime}(\zeta)\right|^{2} N_{q^{\prime}, \varphi}(\zeta) d A(\zeta)=0 .
$$

We conclude that there exists $r \in(0,1)$ such that for all $q, q^{\prime} \in \mathbb{D}$

$$
\int_{\mathbb{D} \backslash D(q, r)}\left|\alpha_{q}^{\prime}(\zeta)\right|^{2} N_{q^{\prime}, \varphi}(\zeta) d A(\zeta)<\frac{k}{4} .
$$

Now, for each $\varepsilon>0, q \in \mathbb{D}$ and $q^{\prime} \in \mathbb{D}$ as in (45), write

$$
\int_{\mathbb{D}}\left|\alpha_{q}^{\prime}(\zeta)\right|^{2} N_{q^{\prime}, \varphi}(\zeta) d A(\zeta)=I_{1}+I_{2}+I_{3}
$$

where

$$
\begin{gathered}
I_{1}=\int_{\mathbb{D} \backslash D(q, r)}\left|\alpha_{q}^{\prime}(\zeta)\right|^{2} N_{q^{\prime}, \varphi}(\zeta) d A(\zeta), \\
I_{2}=\int_{D(q, r) \backslash G_{\varepsilon, q^{\prime}, q}}\left|\alpha_{q}^{\prime}(\zeta)\right|^{2} N_{q^{\prime}, \varphi}(\zeta) d A(\zeta),
\end{gathered}
$$

and

$$
I_{3}=\int_{G_{\varepsilon, q^{\prime}, q} \cap D(q, r)}\left|\alpha_{q}^{\prime}(\zeta)\right|^{2} N_{q^{\prime}, \varphi}(\zeta) d A(\zeta) .
$$


By (46),

$$
I_{1}<\frac{k}{4}
$$

Next,

$$
\begin{aligned}
I_{2} & \leq \varepsilon \int_{D(q, r) \backslash G_{\varepsilon, q^{\prime}, q}}\left|\alpha_{q}^{\prime}(\zeta)\right|^{2}\left(1-\left|\alpha_{q}(\zeta)\right|^{2}\right) d A(\zeta) \\
& \leq \varepsilon\left\|\alpha_{q}\right\|_{*}^{2} \\
& \leq C \varepsilon .
\end{aligned}
$$

By (38)

$$
\begin{aligned}
I_{3} & =\int_{G_{\varepsilon, q^{\prime}, q^{\prime}} \cap D(q, r)}\left|\alpha_{q}^{\prime}(\zeta)\right|^{2} N_{q^{\prime}, \varphi}(\zeta) d A(\zeta) \\
& \leq C \int_{G_{\varepsilon, q^{\prime}, q} \cap D(q, r)}\left|\alpha_{q}^{\prime}(\zeta)\right|^{2}\left(1-\left|\alpha_{\varphi\left(q^{\prime}\right)}(\zeta)\right|^{2}\right) d A(\zeta) \\
& \leq C \int_{G_{\varepsilon, q^{\prime}, q} \cap D(q, r)} \frac{\left(1-|q|^{2}\right)^{2}}{|1-\bar{q} \zeta|^{4}} d A(\zeta) \\
& \asymp \int_{G_{\varepsilon, q^{\prime}, q} \cap D(q, r)} \frac{1}{|D(q, r)|} d A(\zeta) \\
& \asymp \frac{\left|G_{\varepsilon, q^{\prime}, q} \cap D(q, r)\right|}{|D(q, r)|} .
\end{aligned}
$$

By (45), (48), (49), (50), we conclude that there exists $r \in(0,1)$ such that for all $w \in \mathbb{D}$,

$$
\frac{k}{4}<\varepsilon+C \frac{\left|G_{\varepsilon, q^{\prime}, q} \cap D(w, r)\right|}{|D(q, r)|}
$$

or

$$
\frac{k}{4}-\varepsilon \leq \frac{\left|G_{\varepsilon, q^{\prime}, q} \cap D(q, r)\right|}{|D(q, r)|} .
$$

Hence, for any $\varepsilon>0$ with $\varepsilon<k / 4$, the set $G_{\varepsilon, q^{\prime}, q}$ satisfies (44). 
Next, assume that there exists an $\varepsilon>0$ and $r \in(0,1)$ such that for all $q \in \mathbb{D}$, there exists $q^{\prime} \in \mathbb{D}$ such that (44) holds. Then, for each fixed $q \in \mathbb{D}$,

$$
\begin{aligned}
\left\|\alpha_{q} \circ \varphi\right\|_{*}^{2} & \geq \int_{G_{\varepsilon}, q^{\prime}, q}\left|\alpha_{q}^{\prime}(\zeta)\right|^{2} N_{q^{\prime}, \varphi}(\zeta) d A(\zeta) \\
& \geq \int_{G_{\varepsilon, q^{\prime}, q} \cap D(q, r)}\left|\alpha_{q}^{\prime}(\zeta)\right|^{2}\left(1-\left|\alpha_{q}(\zeta)\right|^{2}\right) d A(\zeta) .
\end{aligned}
$$

By $(7),(8)$

$$
\left\|\alpha_{q} \circ \varphi\right\|_{*} \geq C \frac{\left|G_{\varepsilon, q^{\prime}, q} \cap D(q, r)\right|}{|D(q, r)|} \asymp 1
$$

and the conclusion follows.

Lastly, since

$$
\frac{\left|G_{\varepsilon, q^{\prime}, q} \cap D(w, r)\right|}{|D(w, r)|} \leq \frac{\left|G_{\varepsilon} \cap D(w, r)\right|}{|D(w, r)|},
$$

and by (45) and (50), it is clear that $G_{\varepsilon}$ satisfies the reverse Carleson condition.

In section III of [20], Luecking characterized when a measurable subset $F$ of $\mathbb{D}$ satisfies the reverse Carleson condition by using pseudohyperbolic disks, disks whose centers lying on the unit circle $\mathbb{T}$ and also using a collection of disks whose centers lie on $\mathbb{D}$. All the arguments remain valid if we replace $F$ with a collection of measurables subsets $F_{q}, q \in \mathbb{D}$ and we obtain the following proposition.

Proposition 3.9. Given a collection of measurable sets $F_{q}, q \in \mathbb{D}$, the following are equivalent:

(A) There exists a constant $\delta>0$ and $r \in(0,1)$ such that for all $q \in \mathbb{D}$ and for all disks $D$ with centers on $\mathbb{T}$, there exists $q^{\prime} \in \mathbb{D}$ such that

$$
\left|F_{q^{\prime}} \cap D\right|>\delta|\mathbb{D} \cap D|
$$


(B) There exists a constant $\delta_{0}>0$ and $\eta \in(0,1)$ such that for all $q \in \mathbb{D}$ there exists $q^{\prime} \in \mathbb{D}$ such that

$$
\left|F_{q^{\prime}} \cap \mathbb{D}(q, \eta(1-|q|))\right|>\delta_{0}|\mathbb{D}(q, \eta(1-|q|))|
$$

(C) There exists a constant $\delta_{1}>0$ and $r \in(0,1)$ such that for all $q \in \mathbb{D}$ there exists $q^{\prime} \in \mathbb{D}$ such that

$$
\left|F_{q^{\prime}} \cap D(q, r)\right|>\delta_{1}|D(q, r)|
$$

Next, we discuss how the main result in [20] can be modified to allow for a reverse Carleson type condition for a collection of measurable sets $F_{q}, q \in \mathbb{D}$. Let $\delta_{0}$ and $\eta$ be fixed and assume (B) in Proposition 3.9 holds. Since $\eta$ is fixed, as Luecking does in [20, page 6], we abbreviate the notation for the $\operatorname{disk} \mathbb{D}(q, \eta(1-|q|))$ with $D(q)$. Let $A^{1}$ denote the space of analytic and Lebesgue integrable functions on $\mathbb{D}$. Given an analytic function $f$ and $\lambda \in(0,1)$ define the set

$$
E_{\lambda}(q)=\{z \in D(q):|f(z)|>\lambda|f(q)|\}
$$

and the operator

$$
B_{\lambda} f(q)=\frac{1}{\left|E_{\lambda}(q)\right|} \int_{E_{\lambda}(q)}|f(z)| d A(z) .
$$

Note Lemma 1, Lemma 2 and Lemma 3 in [20]. Moreover as done in [20, page 9], if $\varepsilon \in(0,1)$ is small enough and

$$
F=\left\{q \in \mathbb{D}:|f(q)| \geq \varepsilon^{3} B_{\lambda} f(q)\right\}
$$

then

$$
\int_{\mathbb{D}}|f(z)| d A(z)<2 \int_{F} \mid f(z) d A(z)
$$


and for all $q \in F$ there exists $q^{\prime} \in \mathbb{D}$ such that

$$
\frac{1}{|D(q)|} \int_{F_{q^{\prime}} \cap D(q)}|f(z)| d A(z) \geq \frac{1}{2} \delta_{0} \lambda|f(q)| .
$$

Integrating the above over $F$ we obtain

$$
\begin{aligned}
\int_{F} \frac{1}{|D(q)|} \int_{F_{q^{\prime}} \cap D(q)}|f(z)| d A(z) d A(q) & \geq \frac{1}{2} \delta_{0} \lambda \int_{F}|f(q)| d A(q) \\
& \geq \frac{1}{4} \delta_{0} \lambda \int_{\mathbb{D}}|f(q)| d A(q)
\end{aligned}
$$

for all $f \in A^{1}$. The theorem below is now an extension of one direction of the main theorem in $[20]$.

Theorem 3.10. Given a collection of measurable subsets of $\mathbb{D}, F_{q}, q \in \mathbb{D}$, and $F$ as in (52), if there exists a constant $\delta_{0}>0$ and $\eta \in(0,1)$ such that for all $q \in \mathbb{D}$ there exists $q^{\prime} \in \mathbb{D}$ such that

$$
\left|F_{q^{\prime}} \cap \mathbb{D}(q, \eta(1-|q|))\right|>\delta_{0}|\mathbb{D}(q, \eta(1-|q|))|
$$

then there exists a constant $C>0$ such that for all $q \in F$ there exists $q^{\prime} \in \mathbb{D}$ such that for all $f \in A^{1}$

$$
\int_{F} \frac{1}{|D(q)|} \int_{F_{q^{\prime}} \cap D(q)}|f(z)| d A(z) d A(q) \geq C \int_{\mathbb{D}}|f(q)| d A(q)
$$

Remark 3.1. The main theorem in [20] as well as Theorem 3.10 remain valid if $|f|$ is replaced with $|f|^{2}$, see [20, page 6]. Moreover, as Luecking indicates in [20, page 10], instead of $d A(z)$ one may use the measure $\left(1-|z|^{2}\right)^{2} d A(z)$, and the main theorem in [20] is valid. The two critical properties of the weight $w(z)=\left(1-|z|^{2}\right)^{2}$ are $w(q) \leq C \inf \{w(z): z \in D(q, \eta)\}$ and that $w\left(\alpha_{q}(z)\right)=\left|\alpha_{q}^{\prime}(z)\right|^{2}\left(1-|z|^{2}\right)^{2}$. Consequently, Theorem 3.10 is also valid for the measure $\left(1-|z|^{2}\right)^{2} d A(z)$.

We are now ready to prove the following. 
Theorem 3.11. Let $\varphi$ be an analytic self-map of $\mathbb{D}$. Then the composition operator $C_{\varphi}: \mathcal{B} \rightarrow B M O A$ is closed range if and only if there exists an $\varepsilon>0$ and $r \in(0,1)$ such that for all $q \in \mathbb{D}$, there exists $q^{\prime} \in \mathbb{D}$ such that

$$
\frac{\left|G_{\varepsilon, q^{\prime}, q} \cap D(q, r)\right|}{|D(q, r)|} \asymp 1
$$

Proof. First, assume that there exists $\varepsilon>0$ and $r \in(0,1)$ such that for all $q \in \mathbb{D}$, there exits $q^{\prime} \in \mathbb{D}$ such that (54) holds. By Proposition 3.9 this is equivalent to the existence of an $\varepsilon>0$, a constant $\delta_{0}>0$ and $\eta \in(0,1)$ such that for all $q \in \mathbb{D}$ there exists $q^{\prime} \in \mathbb{D}$ such that

$$
\left|G_{\varepsilon, q^{\prime}, q} \cap D(q)\right|>\delta_{0}|D(q)|
$$

Given such $q \in \mathbb{D}$ and $q^{\prime} \in \mathbb{D}$, for each $f \in \mathcal{B}$

$$
\begin{aligned}
\left\|C_{\varphi} f\right\|_{*}^{2} & \geq \int_{G_{\varepsilon, q^{\prime}, q}}\left|f^{\prime}(\zeta)\right|^{2} N_{q^{\prime}, \varphi}(\zeta) d A(\zeta) \\
& \geq \varepsilon \int_{G_{\varepsilon, q^{\prime}, q}}\left|f^{\prime}(\zeta)\right|^{2}\left(1-\left|\alpha_{q}(\zeta)\right|^{2}\right) d A(\zeta) \\
& \geq \varepsilon \int_{G_{\varepsilon, q^{\prime}, q} \cap D(q)}\left|f^{\prime}(\zeta)\right|^{2}\left(1-\left|\alpha_{q}(\zeta)\right|^{2}\right) d A(\zeta)
\end{aligned}
$$

If $\zeta \in D(q)$ then $\zeta \in D(q, r)$, for any fixed $\eta$ and $r \in(0,1)$ with $r \geq 2 \eta / 1+\eta^{2}$, and by (2), (7), (8), $\left(1-\left|\alpha_{q}(\zeta)\right|^{2}\right) \asymp 1$; therefore

$$
\left\|C_{\varphi} f\right\|_{*}^{2} \geq C \int_{G_{\varepsilon, q^{\prime}, q} \cap D(q)}\left|f^{\prime}(\zeta)\right|^{2} d A(\zeta) .
$$

By (7), (55), Theorem 3.10, and Remark 3.1, for all $f \in A^{2}$

$$
\int_{F} \int_{G_{\varepsilon, q^{\prime}, q} \cap D(q)}\left|f^{\prime}(\zeta)\right|^{2} d A(\zeta) d A(q) \geq C \int_{\mathbb{D}}\left|f^{\prime}(q)\right|^{2}\left(1-|q|^{2}\right)^{2} d A(q)
$$


Therefore by (56), for all $f \in A^{2}$

$$
\begin{aligned}
\left\|C_{\varphi} f\right\|_{*}^{2} & \geq C \int_{\mathbb{D}}\left|f^{\prime}(q)\right|^{2}\left(1-|q|^{2}\right)^{2} d A(q) \\
& \asymp\|f\|_{A^{2}}^{2} .
\end{aligned}
$$

Since the $B M O A$ seminorm is invariant under Mobius automorphisms, for all $q \in \mathbb{D}$

$$
\left\|C_{\varphi} f\right\|_{*}^{2} \geq C\left\|f \circ \alpha_{q}-f(q)\right\|_{A^{2}}^{2} .
$$

By (9) we conclude that for all $f \in \mathcal{B},\left\|C_{\varphi} f\right\|_{*} \geq C\|f\|_{\mathcal{B}}$ and by Proposition 3.5, $C_{\varphi}: \mathcal{B} \rightarrow B M O A$ is closed range. Conversely, if $C_{\varphi}: \mathcal{B} \rightarrow B M O A$ is closed range then for all $q \in \mathbb{D},\left\|\alpha_{q} \circ \varphi\right\|_{*} \geq C\left\|\alpha_{q}\right\|_{\mathcal{B}} \geq C$ and by Theorem 3.8 the conclusion follows.

Theorem 3.12. The composition operator $C_{\varphi}$ is closed range on BMOA if and only if there exists a constant $k>0$ such that $\left\|\alpha_{q} \circ \varphi\right\|_{*} \geq k$, for all $q \in \mathbb{D}$.

Proof. First, assume that there exists a constant $k>0$ such that $\left\|\alpha_{q} \circ \varphi\right\|_{*} \geq k$. Then given a $q \in \mathbb{D}$, there exists $q_{n} \in \mathbb{D}$ such that

$$
k^{2} \leq \lim _{n \rightarrow \infty} \int_{\mathbb{D}}\left|\alpha_{q}^{\prime}(\zeta)\right|^{2} N_{q_{n}, \varphi} d A(\zeta)
$$

or

$$
\lim _{n \rightarrow \infty} \int_{\mathbb{D}}\left|\alpha_{q}^{\prime}(\zeta)\right|^{2}\left(k^{2}-N_{q_{n}, \varphi}(\zeta)\right) d A(\zeta) \leq 0 .
$$

Therefore

$$
\lim _{n \rightarrow \infty} \int_{\mathbb{D} \backslash G_{k^{2}, q_{n}, q}}\left|\alpha_{q}^{\prime}(\zeta)\right|^{2}\left(k^{2}\left(1-\left|\alpha_{q}(\zeta)\right|^{2}\right)-N_{q_{n}, \varphi}(\zeta)\right) d A(\zeta) \leq 0
$$

If for each $q \in \mathbb{D}$ there exists a natural number $n$ such that the Lebesgue area measure 
of $\mathbb{D} \backslash G_{k^{2}, q_{n}, q}$ is 0 , then

$$
\begin{aligned}
\left\|C_{\varphi} f\right\|_{*}^{2} & \geq \int_{G_{k^{2}, q_{n}, q}}\left|f^{\prime}(\zeta)\right|^{2} N_{q_{n}, \varphi}(\zeta) d A(\zeta) \\
& =\int_{\mathbb{D}}\left|f^{\prime}(\zeta)\right|^{2} N_{q_{n}, \varphi}(\zeta) d A(\zeta) \\
& \geq k^{2} \int_{\mathbb{D}}\left|f^{\prime}(\zeta)\right|^{2}\left(1-\left|\alpha_{q}(\zeta)\right|^{2}\right) d A(\zeta)
\end{aligned}
$$

and $\left\|C_{\varphi} f\right\|_{*} \geq k\|f\|_{*}$ for all $f \in B M O A$. By Proposition 3.5, $C_{\varphi}$ is closed range on $B M O A$.

Therefore from now on we may assume that for some $q \in \mathbb{D}$ and for all $n$ the Lebesgue area measure of $\mathbb{D} \backslash G_{k^{2}, q_{n}, q}$ is positive. The integrand in (58) is non negative in $\mathbb{D} \backslash G_{k^{2}, q_{n}, q}$, so we must have that for almost every $\zeta \in \mathbb{D} \backslash G_{k^{2}, q_{n}, q}$,

$$
k^{2}\left(1-\left|\alpha_{q}(\zeta)\right|^{2}\right)-N_{q_{n}, \varphi}(\zeta) \rightarrow 0
$$

For each $f \in B M O A$

$$
\int_{\mathbb{D} \backslash G_{k^{2}, q_{n}, q}}\left|f^{\prime}(\zeta)\right|^{2} k^{2}\left(1-\left|\alpha_{q}(\zeta)\right|^{2}\right)-N_{q_{n}, \varphi}(\zeta) d A(\zeta) \leq k^{2}\|f\|_{*}^{2}
$$

therefore by (59) and the Lebesgue Dominated Convergence Theorem

$$
\lim _{n \rightarrow \infty} \int_{\mathbb{D} \backslash G_{k^{2}, q_{n}, q}}\left|f^{\prime}(\zeta)\right|^{2} k^{2}\left(1-\left|\alpha_{q}(\zeta)\right|^{2}\right)-N_{q_{n}, \varphi}(\zeta) d A(\zeta)=0
$$

Write

$$
\int_{\mathbb{D}}\left|f^{\prime}(\zeta)\right|^{2} k^{2}\left(1-\left|\alpha_{q}(\zeta)\right|^{2}\right) d A(\zeta)=I+I I
$$


where

$$
I=\int_{\mathbb{D} \backslash G_{k^{2}, q_{n}, q}}\left|f^{\prime}(\zeta)\right|^{2} k^{2}\left(1-\left|\alpha_{q}(\zeta)\right|^{2}\right) d A(\zeta)
$$

and

$$
I I=\int_{G_{k^{2}, q_{n}, q}}\left|f^{\prime}(\zeta)\right|^{2} k^{2}\left(1-\left|\alpha_{q}(\zeta)\right|^{2}\right) d A(\zeta) .
$$

Note that $I=I_{1}+I_{2}$, where

$$
I_{1}=\int_{\mathbb{D} \backslash G_{k^{2}, q_{n}, q}}\left|f^{\prime}(\zeta)\right|^{2} k^{2}\left(1-\left|\alpha_{q}(\zeta)\right|^{2}\right)-N_{q_{n}, \varphi}(\zeta) d A(\zeta)
$$

and

$$
I_{2}=\int_{\mathbb{D} \backslash G_{k^{2}, q_{n}, q}}\left|f^{\prime}(\zeta)\right|^{2} N_{q_{n}, \varphi}(\zeta) d A(\zeta) .
$$

By $(60), I_{1} \rightarrow 0$ as $n \rightarrow \infty$, and

$$
I I \leq \int_{G_{k^{2}, q_{n}, q}}\left|f^{\prime}(\zeta)\right|^{2} N_{q_{n}, \varphi}(\zeta) d A(\zeta)
$$

Therefore by $(61)$ and for all $q \in \mathbb{D}$

$$
\begin{aligned}
\int_{\mathbb{D}}\left|f^{\prime}(\zeta)\right|^{2} k^{2}\left(1-\left|\alpha_{q}(\zeta)\right|^{2}\right) d A(\zeta) & \leq \int_{\mathbb{D}}\left|f^{\prime}(\zeta)\right|^{2} N_{q_{n}, \varphi}(\zeta) d A(\zeta) \mid+I_{1} \\
& \leq\left\|C_{\varphi} f\right\|_{*}^{2}+I_{1} .
\end{aligned}
$$

We conclude that $k\|f\|_{*} \leq\left\|C_{\varphi} f\right\|_{*}$ for all $f \in B M O A$, and by Proposition $3.5, C_{\varphi}$ is closed range on $B M O A$.

For the converse if $C_{\varphi}$ is closed range on $B M O A$ then $C_{\varphi}$ is bounded below on 
$B M O A$. By Proposition 3.5 and by (21) there exists $\delta>0$ such that for all $q \in \mathbb{D}$,

$$
\begin{aligned}
\left\|\alpha_{q} \circ \varphi\right\|_{*} & \geq \delta\left\|\alpha_{q}\right\|_{*} \\
& \asymp 1,
\end{aligned}
$$

and the conclusion follows.

It is proved in [4, Theorem 12] that $C_{\varphi}$ is bounded in $V M O A$ if and only if $\varphi \in V M O A$. The following now is an immediate corollary of Theorem 3.12.

Corollary 3.13. Let $\varphi \in V M O A$. Then $C_{\varphi}$ is closed range on $V M O A$ if and only if there exists a constant $k>0$ such that $\left\|\alpha_{q} \circ \varphi\right\|_{*} \geq k$, for all $q \in \mathbb{D}$.

Theorem 3.14. A subset $H$ of $\mathbb{D}$ satisfies the reverse Carleson condition if and only if $H$ is a sampling set for BMOA.

Proof. First, assume that $H$ satisfies the reverse Carleson condition. If $H$ is not a sampling set for $B M O A$, then there exists a sequence $\left(f_{n}\right)$ in $B M O A$ such that $\left\|f_{n}\right\|_{*}=1$ and

$$
\lim _{n \rightarrow \infty} \sup _{q^{\prime} \in \mathbb{D}} \int_{H}\left|f_{n}^{\prime}(z)\right|^{2}\left(1-\left|\alpha_{q^{\prime}}(z)\right|^{2}\right) d A(z)=0,
$$

and so for $q^{\prime}=0$,

$$
\lim _{n \rightarrow \infty} \int_{H} \mid\left(\left.f_{n}^{\prime}(\zeta)\right|^{2}\left(1-|\zeta|^{2}\right) d A(\zeta)=0\right.
$$

Since $B M O A$ is continuously contained in $H^{2}$, there exists a constant $C>0$ such that $\left\|f_{n}\right\|_{H^{2}} \leq C$ and by (63), $H$ does not satisfy reverse Carleson condition. We conclude that $H$ is a sampling set for the $B M O A$ space.

Conversely, suppose that $H$ is a sampling set for the $B M O A$ space. Then for all $q \in \mathbb{D}$, 
$\left\|\alpha_{q}\right\|_{*} \asymp 1$ and

$$
\sup _{q^{\prime} \in \mathbb{D}} \int_{H}\left|\alpha_{q}^{\prime}(z)\right|^{2}\left(1-\left|\alpha_{q^{\prime}}(z)\right|^{2}\right) d A(z) \asymp 1 .
$$

Then, there exists $C>0$ such that for all $q \in \mathbb{D}$

$$
\int_{H}\left|\alpha_{q}^{\prime}(z)\right|^{2} d A(z) \geq C
$$

By [16, Theorem 4.1] and (64) we conclude that $H$ satisfies the reverse Carleson condition.

Proposition 3.15. Let $H \subseteq \mathbb{D}$ and $q \in \mathbb{D}$. Then $H$ satisfies the reverse Carleson condition if and only if $\alpha_{q}(H)$ satisfies the reverse Carleson condition.

Proof. For each $H \subseteq \mathbb{D}$ be a Carleson set for the Bergman space, for fixed $q \in \mathbb{D}$, and by making below the change of variables $z=\alpha_{q}(\zeta)$ we obtain

$$
\int_{\alpha_{q}(H)}\left|\alpha_{p}^{\prime}(z)\right|^{2} d A(\zeta)=\int_{H}\left|\left(\alpha_{p} \circ \alpha_{q}\right)^{\prime}(\zeta)\right|^{2} d A(\zeta) .
$$

By [16, Theorem 4.1] the result follows.

Corollary 3.16. Let $\varphi$ be an analytic self-map of $\mathbb{D}$. If $C_{\varphi}$ is closed range on the Bloch space, then it is also closed range on BMOA.

Proof. By [3, Corollary 2.3], $C_{\varphi}$ is closed range on the Bloch space if and only if for all $q \in \mathbb{D},\left\|\alpha_{q} \circ \varphi\right\|_{\mathcal{B}} \asymp 1$. Since $B M O A$ is continuously contained in $\mathcal{B}$, for all $q \in \mathbb{D}$ we have that $\left\|\alpha_{q} \circ \varphi\right\|_{*} \asymp 1$. By Theorem 3.12 we conclude that $C_{\varphi}$ is closed range on BMOA.

Corollary 3.17. Let $\varphi$ be a univalent self-map of $\mathbb{D}$. Then, $C_{\varphi}$ is closed range on $\mathcal{B}$, if and only if $C_{\varphi}$ is closed range on BMOA.

Proof. It is shown in [15, Corollary 2] that for $\varphi$ a univalent self-map of $\mathbb{D}$, if $C_{\varphi}$ is closed 
range on $B M O A$, then it is also closed range on $\mathcal{B}$. By Corollary 3.16 the result is now clear.

Corollary 3.18. Let $\varphi$ be an analytic self-map of $\mathbb{D}$. If $C_{\varphi}$ is closed range on $H^{2}$, then it is also closed range on $B M O A$.

Proof. Given $q \in \mathbb{D}$, let $k_{q}$ denote the normalized reproducing kernel in $H^{2}$, that is

$$
k_{q}(z)=\frac{1-|q|^{2}}{1-\bar{q} z}
$$

It is known, see [22] and [16], that $C_{\varphi}$ is closed range on $H^{2}$ if and only if for all $q \in \mathbb{D}$, $\left\|k_{q} \circ \varphi\right\|_{H^{2}} \asymp 1$. By (12) it is easy to see that $\left\|k_{q} \circ \varphi\right\|_{H^{2}} \asymp|q|\left\|\alpha_{q} \circ \varphi\right\|_{H^{2}}$, if $q \neq 0$. By Theorem 3.12 and since the inclusion map $i: B M O A \rightarrow H^{2}$ is continuous, the conclusion easily follows.

Laitila characterized in [18] the isometries among composition operators on $B M O A$. Moreover he showed that if $\varphi$ is an inner function then $\left\|C_{\varphi} f\right\|_{*}=\|f\|_{*}$, for all $f \in B M O A$. Below we give another characterization of closed range composition operators on $B M O A$. Our proof extends and simplifies the proof in [18] for the isometries.

Theorem 3.19. For each $\varphi$ analytic self map of $\mathbb{D}$, the following conditions are equivalent:

(a) There exists $k \in(0,1]$ such that for every $w \in \mathbb{D},\left\|\alpha_{w} \circ \varphi\right\|_{*} \geq k$.

(b) There exists $k \in(0,1]$ such that for every $w \in \mathbb{D}$ there exists $w^{\prime} \in \mathbb{D}$ with $\left|\alpha_{w}\left(w^{\prime}\right)\right|^{2} \leq 1-k^{2}$, there exists a sequence $\left(q_{n}\right)$ in $\mathbb{D}$ such that $\varphi\left(q_{n}\right) \rightarrow w^{\prime}$ and

$$
\lim _{n \rightarrow \infty}\left\|\varphi_{q_{n}}\right\|_{H^{2}} \geq k
$$

where for all natural numbers $n, \varphi_{q_{n}}=\alpha_{\varphi\left(q_{n}\right)} \circ \varphi \circ \alpha_{q_{n}}$.

Proof. First, assume that (a) holds for some $k \in(0,1]$ and let $w \in \mathbb{D}$. Then, there exists a 
sequence $\left(q_{n}\right)$ in $\mathbb{D}$ such that for all $n$,

$$
\left\|\alpha_{w} \circ \varphi \circ \alpha_{q_{n}}-\alpha_{w}\left(\varphi\left(q_{n}\right)\right)\right\|_{H^{2}}^{2} \geq k^{2}-\frac{1}{n} .
$$

Since $\left(\varphi\left(q_{n}\right)\right)$ is a bounded sequence in $\mathbb{D}$, it has a convergent subsequence in $\overline{\mathbb{D}}$. Without loss of generality, there exists $w^{\prime} \in \overline{\mathbb{D}}$ such that $\varphi\left(q_{n}\right) \rightarrow w^{\prime}$. By (65),

$$
\begin{aligned}
\left|\alpha_{w}\left(\varphi\left(q_{n}\right)\right)\right|^{2} & =\left\|\alpha_{w} \circ \varphi \circ \alpha_{q_{n}}\right\|_{H^{2}}^{2}-\left\|\alpha_{w} \circ \varphi \circ \alpha_{q_{n}}-\alpha_{w}\left(\varphi\left(q_{n}\right)\right)\right\|_{H^{2}}^{2} \\
& \leq 1-k^{2}+\frac{1}{n}
\end{aligned}
$$

therefore, $\left|\alpha_{w}\left(w^{\prime}\right)\right|^{2} \leq 1-k^{2}$ and $w^{\prime} \in \mathbb{D}$.

Let $\alpha_{w} \circ \alpha_{\varphi\left(q_{n}\right)}=\lambda_{n} \alpha_{b_{n}}$ where $\lambda_{n} b_{n}=\alpha_{w}\left(\varphi\left(q_{n}\right)\right)$ and $\left|\lambda_{n}\right|=1$. Then for all $z \in \mathbb{D}$,

$$
\begin{aligned}
\alpha_{w} \circ \varphi \circ \alpha_{q_{n}}(z)-\alpha_{w}\left(\varphi\left(q_{n}\right)\right) & =\alpha_{w} \circ \alpha_{\varphi\left(q_{n}\right)} \circ \varphi_{q_{n}}(z)-\alpha_{w}\left(\varphi\left(q_{n}\right)\right) \\
& =\lambda_{n} \alpha_{b_{n}} \circ \varphi_{q_{n}}(z)-\lambda_{n} b_{n} \\
& =\lambda_{n} \frac{\left(\left|b_{n}\right|^{2}-1\right) \varphi_{q_{n}}(z)}{1-\overline{b_{n}} \varphi_{q_{n}}(z)} .
\end{aligned}
$$

Therefore,

$$
\left\|\alpha_{w} \circ \varphi \circ \alpha_{q_{n}}-\alpha_{w}\left(\varphi\left(q_{n}\right)\right)\right\|_{H^{2}} \leq\left(1+\left|b_{n}\right|\right)|| \varphi_{q_{n}} \|_{H^{2}}
$$

by (65) we conclude, by taking a further subsequence if necessary, that

$$
\begin{aligned}
\lim _{n \rightarrow \infty}\left\|\varphi_{q_{n}}\right\|_{H^{2}} & \geq \frac{k}{1+\left|a_{w}\left(w^{\prime}\right)\right|} \\
& \geq \frac{k}{2}
\end{aligned}
$$


and (b) holds. Note that if $k=1$ above then, $w=w^{\prime}$ and

$$
\lim _{n \rightarrow \infty}\left\|\varphi_{q_{n}}\right\|_{H^{2}}=1
$$

Next, assume that (b) holds for some $k \in(0,1]$ and let $w \in \mathbb{D}$. Then there exists $w^{\prime} \in \mathbb{D}$ with $\left|\alpha_{w}\left(w^{\prime}\right)\right|^{2} \leq 1-k^{2}$, there exists a sequence $\left(q_{n}\right)$ in $\mathbb{D}$ such that $\varphi\left(q_{n}\right) \rightarrow w^{\prime}$ and

$$
\lim _{n \rightarrow \infty}\left\|\varphi_{q_{n}}\right\|_{H^{2}} \geq k
$$

Let $\left|\lambda_{n}\right|=1$ and $\left(b_{n}\right)$ be as in the proof of $(a) \rightarrow(b)$. Then (66) is valid, and for every $n \in \mathbb{N}$

$$
\left\|\alpha_{w} \circ \varphi \circ \alpha_{q_{n}}-\alpha_{w}\left(\varphi\left(q_{n}\right)\right)\right\|_{H^{2}}^{2} \geq\left(1-\left|b_{n}\right|\right)^{2}\left\|\varphi_{q_{n}}\right\|_{H^{2}}^{2}
$$

We conclude that

$$
\begin{aligned}
\left\|\alpha_{w} \circ \varphi\right\|_{*}^{2} & \geq\left\|\alpha_{w} \circ \varphi \circ \alpha_{q_{n}}-\alpha_{w}\left(\varphi\left(q_{n}\right)\right)\right\|_{H^{2}}^{2} \\
& \geq\left(1-\left|b_{n}\right|\right)^{2}\left\|\varphi_{q_{n}}\right\|_{H^{2}}^{2}
\end{aligned}
$$

and by (68)

$$
\left\|\alpha_{w} \circ \varphi\right\|_{*}^{2} \geq \frac{\left(1-\left|\alpha_{w}\left(w^{\prime}\right)\right|^{2}\right)^{2}}{\left(1+\left|\alpha_{w}\left(w^{\prime}\right)\right|\right)^{2}} k^{2} .
$$

If $k \in(0,1)$ and since $\left|\alpha_{w}\left(w^{\prime}\right)\right|^{2} \leq 1-k^{2}$ we conclude by (69) that for all $w \in \mathbb{D}$

$$
\left\|\alpha_{w} \circ \varphi\right\|_{*} \geq \frac{k^{4}}{4}
$$

If $k=1$, then $w=w^{\prime}$ and by (69) $\left\|\alpha_{w} \circ \varphi\right\|_{*}^{2}=1$. The proof is now complete. 


\section{Composition operators on $Q_{p}$}

In this chapter we study the spaces $Q_{p}$ and closed range composition operators on them.

Definition 4.1. For $p \in[0, \infty)$ we say that a function $f$ analytic on $\mathbb{D}$ belongs to $Q_{p}$ if

$$
\|f\|_{Q_{p}}^{2}=\sup _{q \in \mathbb{D}} \int_{\mathbb{D}}\left|f^{\prime}(z)\right|^{2}\left(1-\left|\alpha_{q}(z)\right|^{2}\right)^{p} d A(z)<\infty
$$

where $\|f\|_{Q_{p}}$ is a seminorm and $\left|\left\|f\left|\left\|\left.\right|_{Q_{p}}=|f(0)|+\right\| f \|_{Q_{p}}\right.\right.\right.$ defines a norm in $Q_{p}$ that makes it a Banach space.

The collection of all $Q_{p}$ spaces includes many familiar spaces.

- if $p=0$ then $Q_{0}$ is the Dirichlet space with an equivalent norm.

- if $p=1$ then $Q_{1}$ is the space $B M O A$.

- if $p>1$ then $Q_{p}$ is the Bloch space with an equivalent norm, see [36, Corollary 1.2.1].

It is known, see [36], that if $0 \leq p<q<\infty$ then $Q_{p} \subseteq Q_{q}$ and the inclusion map is continuous. Moreover, if $0 \leq p \neq q \leq 1$ then $Q_{p} \neq Q_{q}$.

Let $p \in(0, \infty)$ and let $f(z)=\sum_{n=0}^{\infty} a_{n} z^{n}$ with $a_{n}$ nonnegative and non increasing. It is shown in [36, Corollary 3.3.1] that $f \in Q_{p}$ if and only if $\sup _{n \in N} n a_{n}<\infty$. For example, $f(z)=\sum_{n=1}^{\infty} \frac{1}{n} z^{n}=\log (1-z) \in Q_{p}$ for all $p$.

Now, we estimate $\left\|\alpha_{q}\right\|_{Q_{p}}$ and show that there exists $c>0$ such that $\left\|\alpha_{q}\right\|_{Q_{p}} \leq c$, for each given $p \geq 0$.

Fixing $p \geq 0, q \in \mathbb{D}$ and by making the change of variables $\alpha_{q}(z)=\zeta$ we obtain,

$$
\begin{aligned}
\left\|\alpha_{q}\right\|_{Q_{p}}^{2} & =\sup _{\lambda \in \mathbb{D}} \int_{\mathbb{D}}\left|\alpha_{q}^{\prime}(z)\right|^{2}\left(1-\left|\alpha_{\lambda}(z)\right|^{2}\right)^{p} d A(z) \\
& =\sup _{\lambda \in \mathbb{D}} \int_{\mathbb{D}}\left(1-\left|\alpha_{\lambda} \circ \alpha_{q}(\zeta)\right|^{2}\right)^{p} d A(\zeta)
\end{aligned}
$$


Since for a fixed $q \in \mathbb{D}$, each Möbius transformation can be written as $e^{i \theta} \alpha_{\lambda} \circ \alpha_{q}$, $\lambda \in \mathbb{D}, \theta \in \mathbb{R}$ we obtain,

$$
\left\|\alpha_{q}\right\|_{Q_{p}}^{2}=\sup _{\lambda \in \mathbb{D}} \int_{\mathbb{D}}\left(1-\left|\alpha_{\lambda}(\zeta)\right|^{2}\right)^{p} d A(\zeta) .
$$

Note that by (2),

$$
\int_{\mathbb{D}}\left(1-\left|\alpha_{\lambda}(\zeta)\right|^{2}\right)^{p} d A(\zeta)=\left(1-|\lambda|^{2}\right)^{p} \int_{\mathbb{D}} \frac{\left(1-|\zeta|^{2}\right)^{p}}{|1-\bar{\lambda} \zeta|^{2 p}} d A(\zeta)
$$

Now we apply [37, Lemma 3.10] with $c=p-2$. We have the following cases:

- If $c<0$ then $0 \leq p<2$ and

$$
\left\|\alpha_{q}\right\|_{Q_{p}}^{2} \lesssim\left(1-|\lambda|^{2}\right)^{p} \lesssim 1
$$

- If $c>0$ then $p>2$ and as $|\lambda| \rightarrow 1$

$$
\left\|\alpha_{q}\right\|_{Q_{p}}^{2} \asymp\left(1-|\lambda|^{2}\right)^{p} \frac{1}{\left(1-|\lambda|^{2}\right)^{p-2}}=\left(1-|\lambda|^{2}\right)^{2} \lesssim 1 .
$$

- if $c=0$ then $p=2$ and as $|\lambda| \rightarrow 1$

$$
\left\|\alpha_{q}\right\|_{Q_{p}}^{2} \asymp \log \frac{1}{1-|\lambda|^{2}}\left(1-|\lambda|^{2}\right)^{2} \lesssim 1
$$

Hence, there exists $c>0$ such that for all $q \in \mathbb{D},\left\|\alpha_{q}\right\|_{Q_{p}}^{2} \leq c$.

For each $p \in[0, \infty)$ the inclusion map $i: Q_{p} \rightarrow \mathcal{B}$ is continuous. Similarly to the proof of (25) we obtain that there exists $c>0$ such that

$$
|f(z)| \leq|f(0)|+c|| f \|_{Q_{p}} \log \frac{1+|z|}{1-|z|} .
$$

Let $p \in[0, \infty)$. The weighted Dirichlet space $\mathcal{D}_{p}$ in the space of all functions $f \in H(\mathbb{D})$ 
satisfying

$$
\|f\|_{\mathcal{D}_{p}}^{2}=\int_{\mathbb{D}}\left|f^{\prime}(z)\right|^{2}\left(1-|z|^{2}\right)^{p} d A(z)<\infty
$$

The above is a seminorm in $\mathcal{D}_{p}$ and $\||f|\|_{\mathcal{D}_{p}}=|f(0)|+\|f\|_{\mathcal{D}_{p}}$ is a norm in $\mathcal{D}_{p}$ and $\mathcal{D}_{p}$ is a Banach space with this norm.

Note that given $q \in \mathbb{D}$ and by making the change of variables $\alpha_{q}(z)=\zeta$ we obtain

$$
\begin{aligned}
\left\|f \circ \alpha_{q}-f(q)\right\|_{\mathcal{D}_{p}}^{2} & =\int_{\mathbb{D}}\left|\left(f \circ \alpha_{q}\right)^{\prime}(z)\right|^{2}\left(1-|z|^{2}\right)^{p} d A(z) \\
& =\int_{\mathbb{D}}\left|f^{\prime}(\zeta)\right|^{2}\left(1-\left|\alpha_{q}(\zeta)\right|^{2}\right)^{p} d A(\zeta)
\end{aligned}
$$

therefore, $f \in Q_{p}$ if and only if

$$
\sup _{q \in \mathbb{D}}\left\|f \circ \alpha_{q}-f(q)\right\|_{\mathcal{D}_{p}}<\infty
$$

Below we show a Lipschitz continuity property that $\left\|f \circ \alpha_{q}-f(q)\right\|_{Q_{p}}$ has with respect to the pseudo hyperbolic metric.

Theorem 4.1. Let $p \in(0,1]$. Then, for each $f \in Q_{p}$ and for all $q, w \in \mathbb{D}$

$$
\left|\left\|f \circ \alpha_{q}-f(q)\right\|_{\mathcal{D}_{p}}^{2}-\left\|f \circ \alpha_{w}-f(w)\right\|_{\mathcal{D}_{p}}^{2}\right| \leq 2^{p+1} \rho(q, w)^{p}\|f\|_{Q_{p}}^{2} .
$$

Proof. Given $p, q \in \mathbb{D}$ and by (5),

$$
\begin{aligned}
A_{p, q} & :=\left.\left|\int_{\mathbb{D}}\right| f^{\prime}(z)\right|^{2}\left(\left(1-\left|\alpha_{q}(z)\right|^{2}\right)^{p}-\left(1-\left|\alpha_{w}(z)\right|^{2}\right)^{p}\right) d A(z) \mid \\
& \leq\left.\int_{\mathbb{D}}\left|f^{\prime}(z)\right|^{2}|| \alpha_{q}(z)\right|^{2}-\left.\left|\alpha_{w}(z)\right|^{2}\right|^{p} d A(z) \\
& \leq 2^{p} \int_{\mathbb{D}}\left|f^{\prime}(z)\right|^{2}|| \alpha_{w}(z)|-| \alpha_{q}(z)||^{p} d A(z) \\
& \leq 2^{p} \rho(w, q)^{p} \int_{\mathbb{D}}\left|f^{\prime}(z)\right|^{2}\left(1-\left|\alpha_{q}(z)\right|\left|\alpha_{w}(z)\right|\right)^{p} d A(z) .
\end{aligned}
$$


Let $\mathcal{A}_{1}:=\left\{z \in \mathbb{D}:\left|\alpha_{w}(z)\right|>\left|\alpha_{q}(z)\right|\right\}$ and $\mathcal{A}_{2}:=\left\{z \in \mathbb{D}:\left|\alpha_{q}(z)\right|>\left|\alpha_{w}(z)\right|\right\}$. Then we have

$$
\begin{aligned}
\int_{A_{1}}\left|f^{\prime}(z)\right|^{2}\left(1-\left|\alpha_{w}(z)\right|\left|\alpha_{q}(z)\right|\right)^{p} d A(z) & \leq \int_{A_{1}}\left|f^{\prime}(z)\right|^{2}\left(1-\left|\alpha_{q}(z)\right|^{2}\right)^{p} d A(z) \\
& \leq\|f\|_{Q_{p}}^{2}
\end{aligned}
$$

and

$$
\begin{aligned}
\int_{A_{2}}\left|f^{\prime}(z)\right|^{2}\left(1-\left|\alpha_{w}(z)\right|\left|\alpha_{q}(z)\right|\right)^{p} d A(z) & \leq \int_{A_{2}}\left|f^{\prime}(z)\right|^{2}\left(1-\left|\alpha_{w}(z)\right|^{2}\right)^{p} d A(z) \\
& \leq\|f\|_{Q_{p}}^{2}
\end{aligned}
$$

Therefore by (72),

$$
A_{p, q} \leq 2^{p+1} \rho(w, q)^{p}\|f\|_{Q_{p}}^{2}
$$

and the conclusion follows.

Remark 4.1. Let $U$ be a measurable subset of $\mathbb{D}, 0<p \leq 1, f \in Q_{p}, w \in \mathbb{D}$ and

$$
A_{w}=\int_{U}\left|f^{\prime}(z)\right|^{2}\left(1-\left|\alpha_{w}(z)\right|^{2}\right)^{p} d A(z)
$$

It is a corollary of the proof of Theorem 4.1 that for each $f \in Q_{p}$ and $q, w \in \mathbb{D}$, $\left|A_{w}-A_{q}\right| \leq 2^{p+1} \rho(w, q)^{p}\|f\|_{Q_{p}}^{2}$.

Since $Q_{1}=B M O A$ the following is part of the above theorem.

Theorem 4.2. There exists $C>0$ such that for each $f \in B M O A$ and $p, q \in \mathbb{D}$

$$
\left|\left\|f \circ \alpha_{p}-f(p)\right\|_{H^{2}}^{2}-\left\|f \circ \alpha_{q}-f(q)\right\|_{H^{2}}^{2}\right| \leq C \rho(p, q)\|f\|_{*}^{2} .
$$


If $p>1$ and $z, \zeta \in \mathbb{D}$ let $\gamma_{\zeta}(z)=1-\left|\alpha_{\zeta}(z)\right|^{2}$. Then for each $q, w, z \in \mathbb{D}$

$$
\left|\gamma_{q}(z)^{p}-\gamma_{w}(z)^{p}\right| \leq p\left(\left|\alpha_{q}(z)\right|^{2}-\left|\alpha_{w}(z)\right|^{2}\right)\left(\gamma_{q}(z)^{p-1}-\gamma_{w}(z)^{p-1}\right)
$$

Therefore, if $f \in Q_{p}$ then similarly to the proof of Theorem 4.1 we obtain the following

Theorem 4.3. Let $p \in(1, \infty)$. Then for each $f \in Q_{p}$ and all $q, w \in \mathbb{D}$

$$
\left|\left\|f \circ \alpha_{q}-f(q)\right\|_{\mathcal{D}_{p}}^{2}-\left\|f \circ \alpha_{w}-f(w)\right\|_{\mathcal{D}_{p}}^{2}\right| \leq 4 p \rho(q, w)\|f\|_{Q_{p}}^{2}
$$

Remark 4.2. Let $U$ be a measurable subset of $\mathbb{D}, p>1, f \in Q_{p}, w \in \mathbb{D}$ and

$$
A_{w}=\int_{U}\left|f^{\prime}(z)\right|^{2}\left(1-\left|\alpha_{w}(z)\right|^{2}\right)^{p} d A(z)
$$

It is a corollary of the proof of Theorem 4.3 that for each $f \in Q_{p}$ and $q, w \in \mathbb{D}$, $\left|A_{w}-A_{q}\right| \leq 4 p \rho(w, q)\|f\|_{Q_{p}}^{2}$.

Definition 4.2. Let $p \in[0, \infty)$. The counting function for $Q_{p}$ for each $q \in \mathbb{D}$, and if $\zeta \in \varphi(\mathbb{D})$ is defined by

$$
N_{q, \varphi}(\zeta, p)=\sum_{\varphi(z)=\zeta}\left(1-\left|\alpha_{q}(z)\right|^{2}\right)^{p}
$$

if $\zeta \notin \varphi(\mathbb{D})$ then $N_{q, \varphi}(\zeta, p)=0$.

From now on we abbreviate the notation for the counting function for $Q_{p}$ to $N_{q, \varphi}(\zeta)$. The Nevanlinna counting function for composition operators in $Q_{p}$ appears with a non-univalent change of variables as is done in [31, page 186]. 
For each $f \in Q_{p}$,

$$
\begin{aligned}
\|f \circ \varphi\|_{Q_{p}}^{2} & =\sup _{q \in \mathbb{D}} \int_{\mathbb{D}}\left|(f \circ \varphi)^{\prime}(z)\right|^{2}\left(1-\left|\alpha_{q}(z)\right|^{2}\right) d A(z) \\
& =\sup _{q \in \mathbb{D}} \int_{\mathbb{D}} \mid\left(\left.f^{\prime}(\varphi(z))\right|^{2}\left|\varphi^{\prime}(z)\right|^{2}\left(1-\left|\alpha_{q}(z)\right|^{2}\right) d A(z),\right.
\end{aligned}
$$

and we have

$$
\|f \circ \varphi\|_{Q_{p}}^{2}=\sup _{q \in \mathbb{D}} \int_{\mathbb{D}}\left|f^{\prime}(\zeta)\right|^{2} N_{q, \varphi}(\zeta) d A(\zeta) .
$$

If $C_{\varphi}$ is a bounded operator on $Q_{p}$ for some $p \in(0, \infty)$ then for all $q \in \mathbb{D},\left\|C_{\varphi} \alpha_{q}\right\|_{Q_{p}} \lesssim 1$ and by (73)

$$
\sup _{q^{\prime} \in \mathbb{D}} \int_{\mathbb{D}}\left|\alpha_{q}^{\prime}(\zeta)\right|^{2} N_{q^{\prime}, \varphi}(\zeta) d A(\zeta) \lesssim 1
$$

By (31) and for all $q^{\prime} \in \mathbb{D}, N_{q^{\prime}, \varphi}(\zeta) d A(\zeta)$ is a collection of uniformly Carleson measures on the Bergman space. By (71) and similarly to the proof of Proposition 3.5 we obtain the following.

Proposition 4.4. Let $p \in(0, \infty)$. The composition operator $C_{\varphi}$ is bounded below on $Q_{p}$ if and only if there exists a $\delta>0$ such that for all $f \in Q_{p},\left\|C_{\varphi} f\right\|_{Q_{p}} \geq \delta\|f\|_{Q_{p}}$.

For each $\epsilon>0$ and $q \in \mathbb{D}$ let $G_{\epsilon, q}$ be

$$
G_{\epsilon, q}=\left\{\zeta: N_{q, \varphi}(\zeta)>\epsilon\left(1-\left|\alpha_{q}(\zeta)\right|^{2}\right)^{p}\right\}
$$

and let $G_{\epsilon}$ be

$$
G_{\epsilon}=\cup_{q \in \mathbb{D}} G_{\epsilon, q}
$$

On the next two theorems we study $C_{\varphi}$ on $Q_{p}$ with $p \in(0,1)$. 
Theorem 4.5. Let $p \in(0,1)$ and assume that $C_{\varphi}$ is a bounded operator on $Q_{p}$. If there exists an $\epsilon>0$ such that

$$
\sup _{q \in \mathbb{D}} \int_{\cap_{q \in \mathbb{D}} G_{\epsilon, q}}\left|f^{\prime}(\zeta)\right|^{2} N_{q, \varphi}(\zeta) d A(\zeta) \asymp\|f\|_{Q_{p}}^{2}
$$

then the composition operator $C_{\varphi}$ is closed range on $Q_{p}$.

Proof. Let $f \in Q_{p}$, assume that (76) holds. Then by (73) and for all $q \in \mathbb{D}$,

$$
\begin{aligned}
\|f \circ \varphi\|_{Q_{p}}^{2} & \geq \sup _{q \in \mathbb{D}} \int_{G_{\varepsilon, q}}\left|f^{\prime}(\zeta)\right|^{2} N_{q, \varphi}(\zeta) d A(\zeta) \\
& \geq \sup _{q \in \mathbb{D}} \int_{\cap_{q \in \mathbb{D}} G_{\epsilon, q}}\left|f^{\prime}(\zeta)\right|^{2} N_{q, \varphi}(\zeta) d A(\zeta) \\
& \geq \epsilon\|f\|_{Q_{p}}^{2}
\end{aligned}
$$

and $C_{\varphi}$ is closed range on $Q_{p}$.

Theorem 4.6. Let $p \in(0,1)$ and assume that $C_{\varphi}$ is a bounded operator on $Q_{p}$. If $C_{\varphi}$ is closed range on $Q_{p}$ then there exists an $\epsilon>0$ such that for all $f \in Q_{p}$

$$
\sup _{q \in \mathbb{D}} \int_{G_{\epsilon}}\left|f^{\prime}(\zeta)\right|^{2} N_{q, \varphi}(\zeta) d A(\zeta) \asymp\|f\|_{Q_{p}}^{2}
$$

Proof. Suppose that $C_{\varphi}$ is closed range on $Q_{p}$. If the conclusion fails, then for every $\varepsilon>0$, $G_{\varepsilon}$ does not satisfy the condition (77) for $Q_{p}$ and we can find a sequence $\left(f_{n}\right)$ in $Q_{p}$ with $f_{n}(0)=0$ and $\left\|f_{n}\right\|_{Q_{p}}=1$ for all $n$ such that

$$
\lim _{n \rightarrow \infty} \sup _{q \in \mathbb{D}} \int_{G_{\frac{1}{n}}}\left|f_{n}^{\prime}(\zeta)\right|^{2} N_{q, \varphi}(\zeta) d A(\zeta)=0 .
$$

For each $n$ we have by (73),

$$
\begin{aligned}
\left\|f_{n} \circ \varphi\right\|_{Q_{p}}^{2} & =\sup _{q \in \mathbb{D}} \int_{\mathbb{D}}\left|f_{n}^{\prime}(\zeta)\right|^{2} N_{q, \varphi}(\zeta) d A(\zeta) \\
& \leq I_{1}+I_{2}
\end{aligned}
$$


where

$$
\begin{gathered}
I_{1}=\sup _{q \in \mathbb{D}} \int_{G_{\frac{1}{n}}}\left|f_{n}^{\prime}(\zeta)\right|^{2} N_{q, \varphi}(\zeta) d A(\zeta), \\
I_{2}=\sup _{q \in \mathbb{D}} \int_{\mathbb{D} \backslash G_{\frac{1}{n}}}\left|f_{n}^{\prime}(\zeta)\right|^{2} N_{q, \varphi}(\zeta) d A(\zeta) .
\end{gathered}
$$

For $n$ a fixed natural number, if $\zeta \notin G_{1 / n}$ then for all $q \in \mathbb{D}$,

$$
N_{q, \varphi}(\zeta) \leq \frac{1}{n}\left(1-\left|\alpha_{q}(\zeta)\right|^{2}\right)^{p}
$$

and

$$
\begin{aligned}
I_{2} & \leq \frac{1}{n} \sup _{q \in \mathbb{D}} \int_{\mathbb{D} \backslash G_{1 / n}}\left|f_{n}^{\prime}(\zeta)\right|^{2}\left(1-\left|\alpha_{q}(\zeta)\right|^{2}\right)^{p} d A(\zeta) \\
& \leq \frac{1}{n} \sup _{q \in \mathbb{D}} \int_{\mathbb{D}}\left|f_{n}^{\prime}(\zeta)\right|^{2}\left(1-\left|\alpha_{q}(\zeta)\right|^{2}\right)^{p} d A(\zeta) \\
& =\frac{1}{n}\left\|f_{n}\right\|_{Q_{p}}^{2} \\
& =\frac{1}{n}
\end{aligned}
$$

By (78), (79), (80) and (82), $\left\|f_{n} \circ \varphi\right\|_{Q_{p}} \rightarrow 0$, as $n \rightarrow \infty$ and $C_{\varphi}$ is not closed range on $Q_{p}$ which contradicts our hypothesis and the conclusion holds.

Theorem 4.7. Let $p>0$ and assume that $C_{\varphi}$ is a bounded operator on $Q_{p}$. Then, there exists $k>0$ such that for all $q \in \mathbb{D},\left\|\alpha_{q} \circ \varphi\right\|_{Q_{p}} \geq k$ if and only if there exists an $\varepsilon>0$ and $r \in(0,1)$ such that for all $q \in \mathbb{D}$, there exists $q^{\prime} \in \mathbb{D}$ such that

$$
\frac{\int_{G_{\varepsilon, q^{\prime}} \cap D(q, r)} N_{q^{\prime}, \varphi}(\zeta) d A(\zeta)}{|D(q, r)|} \asymp 1 .
$$

Proof. First, assume that there exists $k>0$ such that for all $q$ in $\mathbb{D},\left\|\alpha_{q} \circ \varphi\right\|_{Q_{p}} \geq k$. Then, 
for each $q \in \mathbb{D}$ there exists a $q^{\prime} \in \mathbb{D}$ such that

$$
\int_{\mathbb{D}}\left|\alpha_{q}^{\prime}(\zeta)\right|^{2} N_{q^{\prime}, \varphi}(\zeta) d A(\zeta) \geq \frac{k}{2}
$$

By Theorem 3.7 and since for all $q^{\prime} \in \mathbb{D}, N_{q^{\prime}, \varphi}(\zeta) d A(\zeta)$ is a collection of uniformly Carleson measures for the Bergman space,

$$
\lim _{r \rightarrow 1} \sup _{q, q^{\prime} \in \mathbb{D}} \int_{\mathbb{D} \backslash D(q, r)}\left|\alpha_{q}^{\prime}(\zeta)\right|^{2} N_{q^{\prime}, \varphi}(\zeta) d A(\zeta)=0 .
$$

We conclude that there exists $r \in(0,1)$ such that for all $q, q^{\prime} \in \mathbb{D}$

$$
\int_{\mathbb{D} \backslash D(q, r)}\left|\alpha_{q}^{\prime}(\zeta)\right|^{2} N_{q^{\prime}, \varphi}(\zeta) d A(\zeta)<\frac{k}{4} .
$$

Now, for each $\varepsilon>0, q \in \mathbb{D}$ and $q^{\prime} \in \mathbb{D}$ as in (84), write

$$
\int_{\mathbb{D}}\left|\alpha_{q}^{\prime}(\zeta)\right|^{2} N_{q^{\prime}, \varphi}(\zeta) d A(\zeta)=I_{1}+I_{2}+I_{3}
$$

where

$$
\begin{aligned}
I_{1} & =\int_{\mathbb{D} \backslash D(q, r)}\left|\alpha_{q}^{\prime}(\zeta)\right|^{2} N_{q^{\prime}, \varphi}(\zeta) d A(\zeta) . \\
I_{2} & =\int_{D(q, r) \backslash G_{\varepsilon, q^{\prime}}}\left|\alpha_{q}^{\prime}(\zeta)\right|^{2} N_{q^{\prime}, \varphi}(\zeta) d A(\zeta),
\end{aligned}
$$

and

$$
I_{3}=\int_{G_{\varepsilon, q^{\prime}} \cap D(q, r)}\left|\alpha_{q}^{\prime}(\zeta)\right|^{2} N_{q^{\prime}, \varphi}(\zeta) d A(\zeta) .
$$


By (85),

$$
I_{1}<\frac{k}{4}
$$

Next,

$$
\begin{aligned}
I_{2} & \leq \varepsilon \int_{D(q, r) \backslash G_{\varepsilon, q^{\prime}}}\left|\alpha_{q}^{\prime}(\zeta)\right|^{2}\left(1-\left|\alpha_{q}(\zeta)\right|^{2}\right)^{p} d A(\zeta) \\
& \leq \varepsilon\left\|\alpha_{q}\right\|_{Q_{p}}^{2} \\
& \leq C \varepsilon
\end{aligned}
$$

By (38)

$$
\begin{aligned}
I_{3} & =\int_{G_{\varepsilon, q^{\prime}} \cap D(q, r)}\left|\alpha_{q}^{\prime}(\zeta)\right|^{2} N_{q^{\prime}, \varphi}(\zeta) d A(\zeta) \\
& \asymp \int_{G_{\varepsilon, q^{\prime}} \cap D(q, r)} \frac{1}{|D(q, r)|} N_{q^{\prime}, \varphi}(\zeta) d A(\zeta) \\
& \asymp \frac{1}{|D(q, r)|} \int_{G_{\varepsilon, q^{\prime}} \cap D(q, r)} N_{q^{\prime}, \varphi}(\zeta) d A(\zeta) .
\end{aligned}
$$

By (84), (87), (88), (89), we conclude that there exists $r \in(0,1)$ such that for all $w \in \mathbb{D}$,

$$
\frac{k}{4}<\varepsilon+C \frac{\int_{G_{\varepsilon, q^{\prime}} \cap D(q, r)} N_{q^{\prime}, \varphi}(\zeta) d A(\zeta)}{|D(q, r)|}
$$

or

$$
\frac{k}{4}-\varepsilon \leq \frac{\int_{G_{\varepsilon, q^{\prime}} \cap D(q, r)} N_{q^{\prime}, \varphi}(\zeta) d A(\zeta)}{|D(q, r)|} .
$$

Hence, for any $\varepsilon>0$ with $\varepsilon<k / 4$, the set $G_{\varepsilon, q^{\prime}}$ satisfies (83).

Next, assume that there exists an $\varepsilon>0$ and $r \in(0,1)$ such that for all $q \in \mathbb{D}$, there 
exists $q^{\prime} \in \mathbb{D}$ such that (83) holds. Then, for each fixed $q \in \mathbb{D}$,

$$
\begin{aligned}
\left\|\alpha_{q} \circ \varphi\right\|_{Q_{p}}^{2} & \geq \int_{G_{\varepsilon}, q^{\prime}}\left|\alpha_{q}^{\prime}(\zeta)\right|^{2} N_{q^{\prime}, \varphi}(\zeta) d A(\zeta) \\
& \geq \int_{G_{\varepsilon, q^{\prime}} \cap D(q, r)}\left|\alpha_{q}^{\prime}(\zeta)\right|^{2} N_{q^{\prime}, \varphi}(\zeta) d A(\zeta) .
\end{aligned}
$$

By $(7),(8)$

$$
\begin{aligned}
\left\|\alpha_{q} \circ \varphi\right\|_{Q_{p}}^{2} & \geq \int_{G_{\varepsilon, q^{\prime}} \cap D(q, r)}\left|\alpha_{q}^{\prime}(\zeta)\right|^{2} N_{q^{\prime}, \varphi}(\zeta) d A(\zeta) \\
& \asymp \frac{1}{|D(q, r)|} \int_{G_{\varepsilon, q^{\prime}} \cap D(q, r)} N_{q^{\prime}, \varphi}(\zeta) \\
& \asymp 1 .
\end{aligned}
$$

and the conclusion follows.

The following is an immediate corollary of the proof of Theorem 3.12.

Theorem 4.8. Let $p>0$ and assume that $C_{\varphi}$ is a bounded operator on $Q_{p}$. The composition operator $C_{\varphi}$ is closed range on $Q_{p}$ if and only if there exists a constant $k>0$ such that $\left\|\alpha_{q} \circ \varphi\right\|_{Q_{p}} \geq k$, for all $q \in \mathbb{D}$.

The following is an immediate corollary of the proof of Theorem 3.19. The whole proof goes through with the Hardy space seminorm $\|.\|_{H^{2}}$ being replaced with the weighted Dirichlet space seminorm $\|.\|_{\mathcal{D}_{p}}$. Note that for isometries among composition operators on $Q_{p}, k=1$ and $w=w^{\prime}$ below. If $C_{\varphi}$ is an isometry on $Q_{p}$ then by $[29$, Thm 2.1] $\varphi(0)=0$ and so we may only look at the seminorms.

Theorem 4.9. Let $p>0$, assume that $C_{\varphi}$ is a bounded operator on $Q_{p}$. The following conditions are equivalent:

(a) There exists $k \in(0,1]$ such that for every $w \in \mathbb{D},\left\|\alpha_{w} \circ \varphi\right\|_{Q_{p}} \geq k$.

(b) There exists $k \in(0,1]$ such that for every $w \in \mathbb{D}$ there exists $w^{\prime} \in \mathbb{D}$ with 
$\left|\alpha_{w}\left(w^{\prime}\right)\right|^{2} \leq 1-k^{2}$, there exists a sequence $\left(q_{n}\right)$ in $\mathbb{D}$ such that $\varphi\left(q_{n}\right) \rightarrow w^{\prime}$ and

$$
\lim _{n \rightarrow \infty}\left\|\varphi_{q_{n}}\right\|_{D_{p}} \geq k
$$

where for all natural numbers $n, \varphi_{q_{n}}=\alpha_{\varphi\left(q_{n}\right)} \circ \varphi \circ \alpha_{q_{n}}$.

If $p \in(1, \infty)$, then $Q_{p}$ is Bloch space $\mathcal{B}$ with an equivalent norm. Therefore, Theorem 4.7, Theorem 4.8 and Theorem 4.9 provide another approach to closed range composition operators on the $\mathcal{B}$. Moreover, note that Theorem 4.9 provides a characterization for isometries among composition operators on $\mathcal{B}$ with norm \|\|$_{Q_{p}}, p>1$. Recall that if $p>0$ then for each $\epsilon>0$ and $q \in \mathbb{D}$ let $G_{\epsilon, q}$ be

$$
G_{\epsilon, q}=\left\{\zeta: N_{q, \varphi}(\zeta)>\epsilon\left(1-\left|\alpha_{q}(\zeta)\right|^{2}\right)^{p}\right\}
$$

Corollary 4.10. Let $\varphi$ be a non-constant analytic self-map of $\mathbb{D}$, and $p>1$. Then, the following are equivalent

(a) The composition operator $C_{\varphi}$ is closed range on $\mathcal{B}$.

(b) For all $q \in \mathbb{D},\left\|\alpha_{q} \circ \varphi\right\|_{\mathcal{B}} \asymp 1$.

(c) There exists an $\epsilon>0$ and $r \in(0,1)$ such that for all $q \in \mathbb{D}$, there exists $q^{\prime} \in \mathbb{D}$ such that

$$
\frac{\int_{G_{\varepsilon, q^{\prime}} \cap D(q, r)} N_{q^{\prime}, \varphi}(\zeta) d A(\zeta)}{|D(q, r)|} \asymp 1 .
$$

(d) There exists $k \in(0,1]$ such that for every $w \in \mathbb{D}$ there exists $w^{\prime} \in \mathbb{D}$ with $\left|\alpha_{w}\left(w^{\prime}\right)\right|^{2} \leq 1-k^{2}$, there exists a sequence $\left(q_{n}\right)$ in $\mathbb{D}$ such that $\varphi\left(q_{n}\right) \rightarrow w^{\prime}$ and

$$
\lim _{n \rightarrow \infty}\left\|\varphi_{q_{n}}\right\|_{D_{p}} \geq k
$$

where for all natural numbers $n, \varphi_{q_{n}}=\alpha_{\varphi\left(q_{n}\right)} \circ \varphi \circ \alpha_{q_{n}}$. 


\section{References}

[1] S, Axler, Bergman spaces and their operators. Surveys of some recent results in operator theory, Vol. I, 1-50, Pitman Res. Notes Math. Ser., 171, Longman Sci. Tech., Harlow, 1988.

[2] J. Akeroyd, P. Ghatage, Closed-range composition operators on $A^{2}$, Illinois J. Math. 52, (2008), no. 2, 533-549.

[3] J. Akeroyd, P. Ghatage, M. Tjani, Closed-range composition operators on $A^{2}$ and the Bloch Space, Integral Equations Operator Theory, 68 (2010), 503-507.

[4] J. Arazy, S.D. Fisher, J. Peetre, Mobius invariant function spaces, J. Reine Angew. Math., 363 (1985), 110-145.

[5] A. Baernstein, Analytic functions of bounded mean oscillation, Aspects of contemporary complex analysis (Proc. NATO Adv. Study Inst., Univ. Durham, Durham, 1979), pages 3-36, Academic Press, London-New York, 1980.

[6] P.S. Bourdon, J.A. Cima and A. Matheson, Compact composition Operators on BMOA, Trans. Amer. Math. Soc. 351, no. 6, 2183-2196.

[7] H. Chen, Boundedness from below of composition operators on the Bloch spaces, Science in China. Series A, Mathematics, 46 (2003), 838-846.

[8] H. Chen, P. Gauthier, Boundedness from below of composition operators on $\alpha$ - Bloch spaces, Canad. Math. Bull. 51, (2008), no. 2, 195-204.

[9] J.A. Cima, J. Thomson, W. Wogen, On some properties of composition operators, Indiana Univ. Math. J. 24, (1974/75), 215-220.

[10] J.A. Cima, G. Schober, Analytic functions with bounded mean oscillation and logarithms of $H^{p}$-functions, Math. Z., 151 (1976), 295-300.

[11] P. Duren, A. Schuster, Bergman Spaces, Math. Surveys Monogr., Vol 100, American Mathematical Society, Providence, RI, 2004.

[12] J.B. Garnett, Bounded Analytic Functions, Revised first edition. Graduate Texts in Mathematics, 236, Springer, New York, 2007.

[13] P. Ghatage, D. Zheng, Analytic functions of bounded mean oscillation and the Bloch space, Integral Equations Operator Theory, 17 (1993), no. 4, 501-515.

[14] P. Ghatage, J. Yan, D. Zheng, Composition Operators with closed range on the Bloch space, Proc. Amer. Math. Soc. 129 (2001), no 7, 2039-2044.

[15] P. Ghatage, D. Zheng, N. Zorboska, Sampling sets and closed range composition Operators on the Bloch Space, Proc. Amer. Math. Soc. 133 (2005), no 5, 1371-1377. 
[16] P. Ghatage, M. Tjani, Closed range composition operators on Hilbert function spaces, J. Math. Anal. Appl. 431 (2015), no. 2, 841-866.

[17] M. Jovovic, B.D MacCluer, Composition operators on Dirichlet spaces, Acta Sci. Math. (Szeged) 63 (1997), no.1-2, 229-247.

[18] J. Laitila, Isometric composition operators on BMOA, Math. Nachr. 283 (2010), no. 11, 1646-1653.

[19] J. Laitila, P.J. Nieminen, E. Saksman, H.O. Tylli, Compact and weakly compact composition operators on BMOA Complex Anal. and Oper. Theory, 7, 2013, 163-181.

[20] D. H. Luecking, Inequalities on Bergman spaces, Illinois J. Math. 25 (1981), 1-11.

[21] D. H. Luecking, Forward and reverse Carleson inequalities for functions in Bergman spaces and their derivatives, Amer. J. Math. 107 (1985), no 1, 85-111.

[22] K. Luery, Composition operators on Hardy spaces of the disk and half-space, University of Florida, Doctoral Dissertation, 2013.

[23] B.D. MacCluer, Elementary functional analysis, Graduate Texts in Mathematics, 253, Springer, New York, 2009.

[24] S. Makhmutov, M. Tjani, Composition operators on some Mbius invariant Banach spaces, Bull. Austral. Math. Soc. 62, (2000), no. 1, 1-19.

[25] L.A. Rubel, R.M. Timoney, An external property of the Bloch space, Proceedings of the American Mathematical Society, 75 (1979), 45-49.

[26] W. Rudin, Real and complex analysis, Third edition, McGraw-Hill Book Co., New York, 1987.

[27] J.V. Ryff, Subordinate $H^{p}$ functions, Duke Math. J., 33, 1966, 347-354.

[28] D. Sarason, Function theory on the unit circle. Notes for lectures given at a Conference at Virginia Polytechnic Institute and State University, Blacksburg, Va., June 19-23, 1978. Virginia Polytechnic Institute and State University, Department of Mathematics, Blacksburg, Va., 1978.

[29] M. Shabazz, M. Tjani, Isometries among composition operators on Besov type spaces, Complex Variables and Elliptic Equations, 61:6, (2015), 761-771.

[30] J. H. Shapiro, The essential norm of a composition operator, Ann. of Math. (2), 125 (1987), no 1, 375-404.

[31] J. H. Shapiro, Composition Operators and Classical Function Theory, Springer-Verlag, New York, 1993.

[32] W. Smith, Compactness of composition operators on BMOA, Proc. Amer. Math. Soc. 127, (1999), no. 9, 2715-2725. 
[33] M. Tjani, Compact composition operators on some Möbius invariant Banach Spaces, Michigan State University, Doctoral Dissertation, (1996).

[34] M. Tjani, Closed range composition operators on Besov type spaces, Complex Anal. Oper. Theory, 8 (2014) no. 1, 189-212.

[35] K. J. Wirths, J. Xiao, Global integral criteria for composition operators, Journal of Mathematical Analysis and Applications. 269 (2002) no. 2, 702-715.

[36] J. Xiao, Holomorphic Q classes. Lecture Notes in Mathematics, 1767, Springer-Verlag, Berlin, 2001.

[37] K. Zhu, Operator Theory in Function Spaces, Second edition, Mathematical Surveys and Monographs, 138, American Mathematical Society, Providence, RI, 2007.

[38] N. Zorboska, Composition operators with closed range, Trans. Amer. Math. Soc. 344 (1994) no. 2, 791-801. 